\title{
The Effect of Foam on Waves and the Aerodynamic Roughness of the Water Surface at High Winds
}

\author{
YU. TROITSKAYA \\ Institute of Applied Physics, Nizhny Novgorod, and A.M. Obukhov Institute of Atmospheric Physics, \\ Moscow, Russia \\ D. SERGEev, A. Kandaurov, And M. VDOVIN \\ Institute of Applied Physics, Nizhny Novgorod, Russia
}

\section{S. ZILITINKEVICH}

Finnish Meteorological Institute, and Institute of Atmospheric and Earth System Research, University of Helsinki, Helsinki, Finland and Faculty of Radio-physics, Lobachevsky State University of Nizhny Novgorod, Nizhny Novgorod, Russia

(Manuscript received 16 August 2018, in final form 5 January 2019)

\begin{abstract}
This paper models the impact of the presence of foam on the short-wave component of surface waves and momentum exchange in the atmospheric boundary layer at high winds. First, physical experiments were carried out in a wind-wave flume in which foam can be artificially produced at the water surface. Tests were conducted under high-wind-speed conditions where equivalent $10-\mathrm{m}$ wind speed ranged from 12 to $38 \mathrm{~m} \mathrm{~s}^{-1}$, with measurements made of the airflow parameters, the frequency-wavenumber spectra of the surface waves, the foam coverage of the water surface, and the distribution of the foam bubbles. Analysis of the resulting data indicates that the surface drag coefficient correlates with the fraction of foam coverage and the mean square slope (MSS) of the water surface, and that, at a certain wind speed, the MSS decreases with an increase in the fraction of foam coverage. Based on these results, we suggest a simple model for eddy viscosity in the turbulent boundary layer over a fractionally foam-covered wave surface. The measurements in a laboratory environment are shown to be in good agreement with the predictions of a quasi-linear model of the atmospheric boundary layer over a waved water surface that adopts this eddy viscosity. Adaptation of the proposed model to field conditions is discussed, and the synergetic effect of foam at the water surface and spray in the marine atmospheric boundary layer on ocean surface resistance at high winds is estimated so as to be able to explain the observed peaking dependence of the surface drag coefficient on the $10-\mathrm{m}$ wind speed.
\end{abstract}

\section{Introduction}

Parameterization of the small-scale air-sea coupling responsible for energy and momentum fluxes between the atmosphere and ocean is crucial for accurately forecasting the trajectory and, especially, the intensity of tropical cyclones. In particular, Emanuel (1986) demonstrated that the intensity of tropical cyclones can be explained in terms of wind-induced surface heat exchange (WISHE), with the cyclone being regarded as a

Corresponding author: Yuliya Troitskaya, yuliya@hydro.appl. sci-nnov.ru
Carnot engine driven by the difference in temperature between the ocean and the top of the troposphere. The energy input to the Carnot cycle is defined by the moist enthalpy flux from the ocean to the atmosphere, and the kinetic energy is mainly dissipated through the action of turbulent fluctuations driven by the air-sea momentum flux or turbulent shear stress (see Bister and Emanuel 1998). More recent improvements in forecasting the intensity of storms have been accomplished through the use of coupled ocean-atmosphere models that take feedback from the ocean into account (see Bender and Ginis 2000; Falkovich et al. 1995). Shear stress at the water surface is the driving force responsible for mixing 
the upper layer and cooling the surface of the ocean, so to reliably forecast the intensity of a storm, it needs to be correctly parameterized for high-wind-speed conditions. It is also crucial for correctly forecasting storm surges. The turbulent shear stress, $T=\rho_{a} u_{*}^{2}$, is usually parameterized with the bulk formula $T=\rho_{\text {air }} C_{D} U_{10}^{2}$, where $\rho_{\text {air }}$ is the air density, $C_{D}$ is the drag coefficient of the sea surface, $U_{10}$ is the wind velocity at the reference height of $10 \mathrm{~m}$ from the ocean surface, and $u_{*}$ is the wind friction velocity.

The surface drag coefficient is a function of wind speed. Numerous measurements (e.g., Garratt 1977; Large and Pond 1981; Taylor and Yelland 2001; Fairall et al. 2003) performed at wind speeds below $25 \mathrm{~m} \mathrm{~s}^{-1}$ show an increase in $C_{D}$ with increase in wind speed. Generally, the increase in $C_{D}$ is associated with a widening of the surface wave spectrum with increasing wind speed and an increase in the surface form drag (see, e.g., Makin et al. 1995; Janssen 1989). Data collected by and parameterization carried out by Foreman and Emeis (2010) and Andreas et al. (2012) indicate that, as wind speed increases, $C_{D}$ shows a tendency to saturation. There is considerable uncertainty about the dependence of $C_{D}$ on $U_{10}$ at winds exceeding $30 \mathrm{~m} \mathrm{~s}^{-1}$. On the one hand, there is strong evidence from field measurements that $C_{D}$ depends on $U_{10}$ nonmonotonically: it reaches a maximum at $U_{10}=35-40 \mathrm{~m} \mathrm{~s}^{-1}$ and then decreases (see Powell et al. 2003; Jarosz et al. 2007; Holthuijsen et al. 2012; Richter et al. 2016). On the other hand, the integral estimates by Bell et al. (2012), which were based on experimental data by Black et al. (2007), show a leveling-off of $C_{D}$ at $U_{10}>50 \mathrm{~m} \mathrm{~s}^{-1}$. Laboratory experiments indicate saturation (see Donelan et al. 2004; Takagaki et al. 2012, 2016) or a tendency to saturation (see Troitskaya et al. 2012) of the surface drag coefficient at hurricane wind speeds.

The theoretical models suggested to explain sea surface drag reduction under hurricane winds exploit either the peculiar properties of the water surface under the force of hurricane wind or the effect of sea spray, the concentration of which in the air boundary layer is enhanced dramatically during storms. For example, Kudryavtsev and Makin (2007) and Kukulka et al. (2007) explain sea surface drag reduction in terms of the characteristics of airflow over breaking waves, as these determine the form drag of the sea surface. Similarly, Donelan et al. (2004) qualitatively explained the stabilization of the drag coefficient at wind speeds above $35 \mathrm{~m} \mathrm{~s}^{-1}$ in terms of the asymmetry of the shape of dominant waves, which have steep front slopes, assuming flow separation from the wave crest. This assumption is based on laboratory experiments by Reul et al. (1999), where airflow separation at the crests of breaking waves was observed in instantaneous velocity patterns obtained using the particle image velocimetry (PIV) method. This explanation is the most appropriate to the case of the initial stage of development of surface waves. A similar mechanism was suggested by Troitskaya and Rybushkina (2008), who explained sea surface drag reduction at hurricane wind speeds as a result of a reduction in the efficiency of wind-wave momentum exchange due to sheltering without separation.

Another approach, which is more appropriate to a mature sea state, exploits the effect of sea spray on windwave momentum exchange. Andreas and Emanuel (2001), Andreas (2004), and Kudryavtsev and Makin (2011) estimated the momentum exchange of sea drops and airflow, while Makin (2005) and Kudryavtsev (2006) focused on the effect of sea drops on the stratification of the air-sea boundary layer, similar to the model of a turbulent boundary layer with suspended particles developed by Barenblatt and Golitsyn (1974). Troitskaya et al. (2017c, 2018b) interpreted the peaking dependence of sea surface drag as resulting from the peculiarities of the momentum flux from the atmosphere to the ocean in the course of sea spray production due to bag-breakup fragmentation of the air-sea interface; this mechanism has been proved to be the dominant mode of generation of spume droplets torn off the crests of surface waves by strong winds (see Troitskaya et al. 2017a,b, 2018a).

Recently, Soloviev et al. (2014) suggested that KelvinHelmholtz instability of the air-sea interface can be used to explain the anomalous surface drag coefficient at hurricane winds. In agreement with data by Powell et al. (2003), Jarosz et al. (2007), Holthuijsen et al. (2012), and Richter et al. (2016) and data assimilation by Bell et al. (2012), this approach predicts that $C_{D}$ will have a special dependence on wind speed where it reaches a maximum at $U_{10}$ of about $35 \mathrm{~m} \mathrm{~s}^{-1}$ and a minimum at $U_{10}$ of about $60 \mathrm{~m} \mathrm{~s}^{-1}$, after which it increases with wind speed.

An alternative explanation of the low aerodynamic resistance of the water surface at high winds is based on the effects of foam, which can make the water surface "slippery" for airflow (Shtemler et al. 2010; Holthuijsen et al. 2012; Golbraikh and Shtemler 2016). By examining aircraft photography, Holthuijsen et al. (2012) determined that at high winds a very limited fraction of the sea surface is covered by white caps composed of dense foam patches formed through wave breaking. For example, at wind speeds up to approximately $50 \mathrm{~m} \mathrm{~s}^{-1}$, this dense foam makes up less than $5 \%$ of the surface. However, at these wind speeds, the wave surface is almost completely covered by less dense foam streaks stretched out in the wind direction. They found that the onset of the leveling off of the drag coefficient and the onset of the creation of streaks coincide at a wind speed 
of about $25 \mathrm{~m} \mathrm{~s}^{-1}$. Furthermore, they found that at about $40 \mathrm{~m} \mathrm{~s}^{-1}$, these streaks merge and there is a decrease in surface roughness. On the basis of these findings, they concluded that foam coverage, including foam streaks, plays a crucial role in the surface drag of the ocean during storms. Following these results, Golbraikh and Shtemler (2016) suggested a parameterization of the aerodynamic roughness of a foam-covered sea surface based on the assumption that the roughness height is prescribed by the average radius of a foam bubble in the foam-covered areas. Using this assumption and data from Holthuijsen et al. (2012) on the fractional coverage by foam and streaks, Golbraikh and Shtemler (2016) demonstrated a dependence of the ocean surface drag on wind speed that is in fair agreement with available observations.

The state of the art thus clearly indicates that foam is an important factor governing air-sea momentum exchange at storm wind speeds. In this study, we investigate the effect of foam on surface waves and the air boundary layer in the controlled environment of a laboratory facility, where the generation of artificial foam and direct measurements of its effect on the wind and wave parameters are possible. The structure of the paper is as follows. In section 2, we describe the experimental setup. Section 3 presents the experimental techniques used for measuring airflow, wave and foam parameters and describes the experimental dependencies obtained. In section 4 , we interpret the experimental data described in section 3 in terms of the quasi-linear model of the turbulent airflow over a waved water surface developed by Troitskaya et al. (2012, 2014). In section 5, we estimate the effect of foam on the surface drag coefficient under field conditions. In section 6 , we estimate the synergetic effect of foam and the phenomenon of bag-breakup fragmentation of the air-sea interface on the aerodynamic roughness of the water surface for wind flows at field conditions.

\section{Experimental setup}

Experiments were performed with the high-speed wind-wave flume in the Large Thermally Stratified Tank at the Institute of Applied Physics, Russian Academy of Sciences [IAP RAS 2018; see details in Troitskaya et al. (2012)] with the overall sizes $20 \mathrm{~m}$ in length, $4 \mathrm{~m}$ in width, and $2 \mathrm{~m}$ in depth (see http://unu. ipfran.ru/pool). The airflow channel has a $0.4 \mathrm{~m} \times 0.4 \mathrm{~m}$ cross section at the water surface and is open at the bottom and at the outlet; the sidewalls are submerged to a depth of $0.35 \mathrm{~m}$. The total length is $10 \mathrm{~m}$, and the centerline velocity range is $3-25 \mathrm{~m} \mathrm{~s}^{-1}$. The tank is filled with freshwater, with the temperature ranging from $15^{\circ}$ to $24^{\circ} \mathrm{C}$. The measured value of the surface tension for freshwater was $\sigma=(7.1 \pm 0.25) \times 10^{-2} \mathrm{Nm}^{-1}$.

By definition, the roughness parameter $z_{0}$ is explicitly related to the coefficient of aerodynamic resistance of the water surface $C_{D}$ reduced to neutral stratification. Indeed, in this case, the wind speed profile is

$$
U(z)=\frac{u_{*}}{\kappa} \ln \frac{z}{z_{0}},
$$

where $u_{*}$ is the wind friction velocity and $\kappa=0.4$ is the von Kármán constant.

The 10-m surface drag coefficient is then

$$
C_{D}=\frac{u_{*}^{2}}{U_{10}^{2}}=\frac{\kappa^{2}}{\ln ^{2}\left(H_{10} / z_{0}\right)} .
$$

Here, $U_{10}$ is the $10-\mathrm{m}$ wind speed, and $H_{10}=10 \mathrm{~m}$.

The experiments investigated the dependence of the surface drag coefficient on the dynamic wind speed and the characteristics of the surface in the presence and absence of foam. The experimental setup is shown in Fig. 1a. A simple foam generator was used to produce artificial stable foam at the water surface (Fig. 1b). The device consists of two diffusers, which are parallel tubes $35 \mathrm{~cm}$ in length and $1 \mathrm{~cm}$ in diameter, and perforated at the side with 1-mm diameter holes with a pitch of $7 \mathrm{~mm}$. The tubes were laid side by side and wrapped in porous rubber. The device was submerged with the water level $0.5 \mathrm{~cm}$ above the upper edge of the porous rubber layer and positioned horizontally, perpendicular to the axis of the channel, $1.2 \mathrm{~m}$ from the airflow inlet. A sodium lauryl sulfate (SLS) solution was ejected under the pressure of a $1.5-\mathrm{m}$ water column from the first diffuser. Compressed air was supplied at a pressure of $1.5 \mathrm{~atm}$ from the second diffuser to push the SLS solution through the layer of foam rubber. As a result, a finely dispersed foam formed at the water surface and was carried away by the wind drift flow. In the course of the experiments, the level of the SLS solution and the pressure of the compressed air were kept constant, ensuring a constant rate of foam production. Special test experiments were performed in which the compressed air was ejected from the nozzle without a corresponding supply of SLS solution. In this regime, bubbles were produced only locally in the vicinity of the diffuser and did not reach the working section. The effect of air bubbles on the surface waves in the working section was found to be negligible (see section 3). In the working regime, both the compressed air and SLS solution were ejected, and foam reached the working section. The shadow photographs of the water surface covered by the foam at two substantially different airflow velocities prescribed by the frequency of the fan are presented 


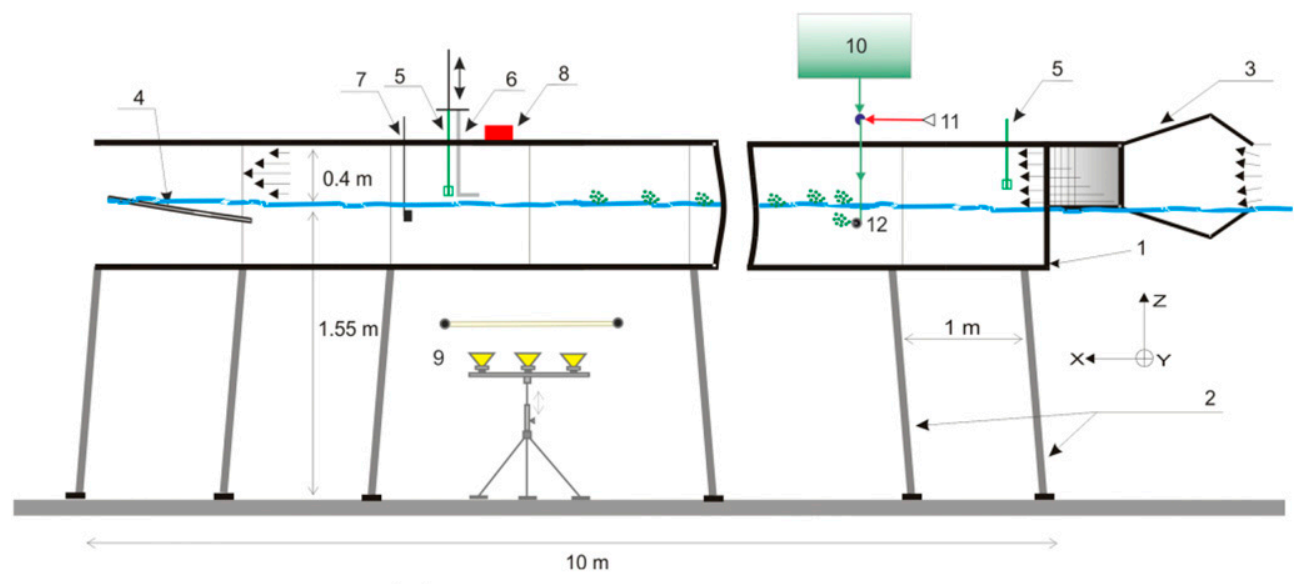

(a)

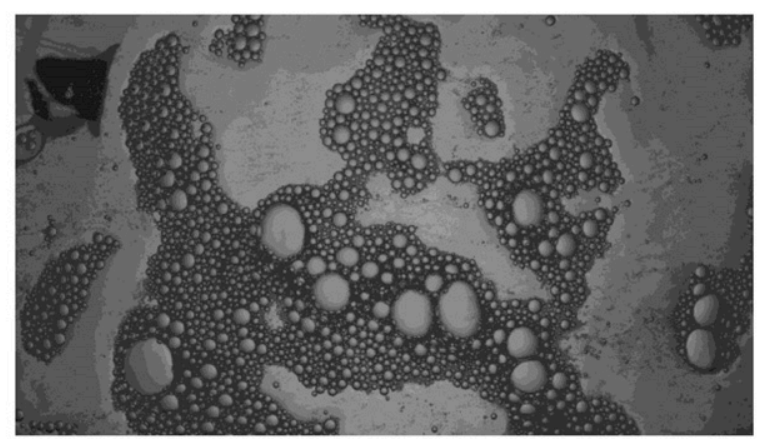

(c)

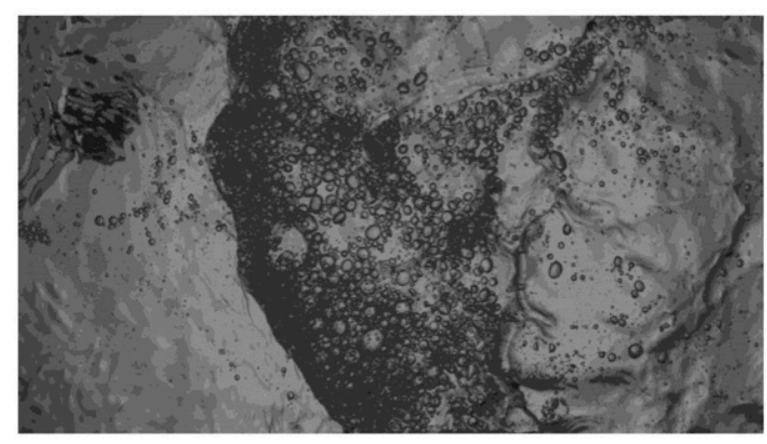

(d)

FIG. 1. (a) Experimental setup: 1, flume; 2, supports; 3, honeycomb; 4, wave-damping beach; 5, hot-film probe; 6 , pitot tube; 7, threechannel wave gauge array; 8 , high-speed camera; 9, illumination system; 10, SLS solution reservoir; 11, compressed-air supply; 12, foam generator. Sizes are in meters. (b) Enhanced cross section of the foam generator. Also shown are shadow photographs of the foam at the water surface for fan rotation frequencies of (c) 20 and (d) $40 \mathrm{~Hz}$.

in Figs. 1c and 1d. Before and after each run in which artificial foam was generated, the parameters of the water in the tank were monitored to assess the possible effect of dissolved SLS not consumed by foam creation. The surface tension coefficient was estimated by the drop method (see Stauffer 1965) and the viscosity was measured with a standard glass viscosimeter. It was found that the concentration of the dissolved SLS was sufficiently low as to not affect the surface tension and the viscosity of the water in the working section at a distance $6.5 \mathrm{~m}$ from the foam generator within the experimental accuracy. In all cases, the maximum deviation of the surface tension was about $0.2 \times 10^{-2} \mathrm{~N} \mathrm{~m}^{-1}$ and of viscosity was $7 \times$ $10^{-4} \mathrm{~cm}^{2} \mathrm{c}^{-1}$ (within the error of measurement). This indicates that the physical properties of the water in the tank were conserved and that dissolved, unused SLS had minimal potential to influence the wind-wave coupling. For comparison, experimental runs with clean water with the foam generator switched off were also performed. Two independent experimental runs were performed in each regime to ensure the validity of the data.

\section{Experimental methods and results}

During the experiments, we measured the airflow parameters (friction velocity $u_{*}, 10-\mathrm{m}$ wind speed $U_{10}$, and surface drag coefficient $C_{D}$ ), the characteristics of the surface waves [frequency $S(\omega)$, wavenumber $S(k)$, spectra, significant wave height $H_{s}$, and mean square slope (MSS)] and the foam parameters (fractional coverage and the size distribution of bubbles). The experimental techniques employed and the data acquired are discussed in this section.

\section{a. Airflow parameters}

The parameters of the airflow boundary layer in the channel were retrieved using the profiling method. 
For this purpose, we measured profiles of the airflow velocity in the working section by using an L-shaped pitot tube with a Baratron MKS 226 A differential pressure transducer, which provided a velocity measurement accuracy of about $3 \mathrm{~cm} \mathrm{~s}^{-1}$. The scanning method was used, with consecutive height increments of 3-5 $\mathrm{mm}$ and accruing time of $60 \mathrm{~s}$ at each point. Two velocity profiles were measured for each fixed wind parameter for subsequent averaging. The lowest level of scanning was $1 \mathrm{~cm}$ from the crests of the waves and depended on the wind speed, while the upper limit was $38 \mathrm{~cm}(2 \mathrm{~cm}$ below the upper lid of the channel). The temperature and wind speed at the inlet of the flume were controlled with a hot film gauge. Another temperature gauge was placed under the water in the working section to measure the temperature of the surface layer. Examples of airflow velocity profiles at different fan rotation frequencies are shown in Fig. 2.

The parameters of the atmospheric boundary layer were determined by implementing the algorithm suggested by Troitskaya et al. (2012). This extends a model of the boundary layer near a flat surface described by Hinze (1959) to the case of channel flow with a waved lower surface. It is well known that the boundary layer on a smooth flat plate comprises a viscous sublayer with a thickness of about $\delta_{\text {vis }}=\nu_{a} / u_{*}$ (less than $\left.1 \mathrm{~mm}\right)$ above a layer with constant fluxes with a thickness of about $0.15 \delta$, where $\delta$ is the boundary layer displacement thickness, and the "wake" part of the turbulent boundary layer, which extends up to the border of the displacement layer. Over a waved surface, an additional layer is generated in the turbulent boundary layer in which there is a transfer of momentum from the airflow to the wave perturbations on the water surface; the sum of the turbulent $\tau_{\text {turb }}(\eta)$ and wave $\tau_{\text {wave }}(\eta)$ momentum fluxes is constant (see. e.g., Makin et al. 1995):

$$
\tau_{\text {turb }}(\eta)+\tau_{\text {wave }}(\eta)=u_{*}^{2} .
$$

Here, $\tau_{\text {wave }}(\eta)$ decreases with increasing distance from the boundary on the scale $h_{\text {wave }}$, which, according to Belcher et al. (1993) can be determined from the following relationship:

$$
k h_{\text {wave }}\left(\frac{\kappa V_{p}}{u_{*}}+\ln k z_{0}-\ln h_{\text {wave }} k\right)=\kappa^{2} .
$$

Here, $V_{p}$ and $k$ are the phase velocity and the wavenumber corresponding to the frequency of the peak of the surface elevation spectrum. The numerical values of the wind and wave parameters in the flume detailed below in Figs. 3 and 4 give an estimate of $k h_{\text {wave }} \sim 0.01$ (see also Kandaurov et al. 2014). Here, the condition

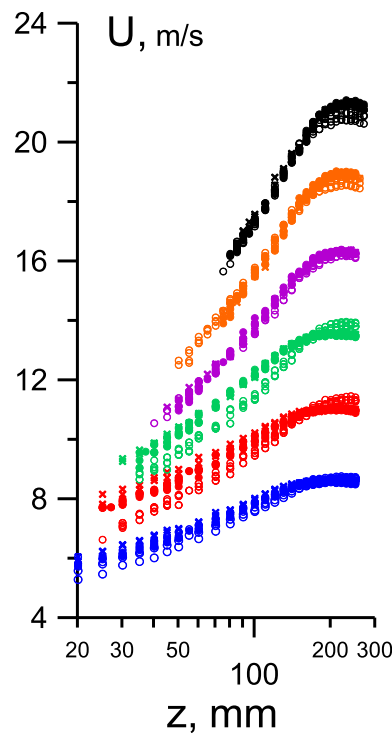

(a)

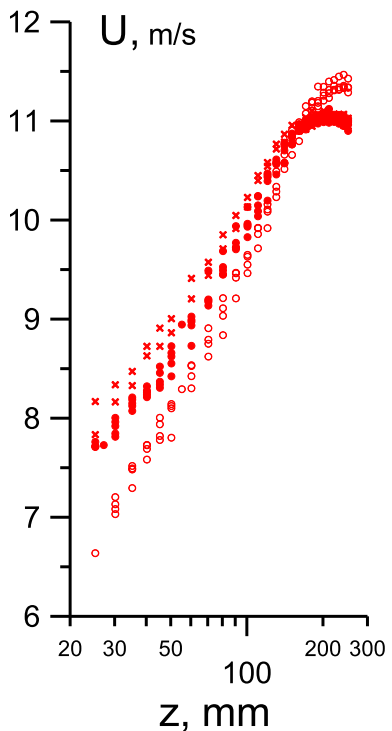

(b)
FIG. 2. (a) Airflow velocity profiles for six fan rotation frequencies: 20 (blue), 25 (red), 30 (green), 35 (purple), 40 (orange), and $45 \mathrm{~Hz}$ (black). Closed symbols indicate clean water, open symbols indicate compressed air and SLS solution supplied to the foam generator, and crosses indicate only compressed air supplied. (b) As in (a), but at an enhanced scale for fan rotation frequency $F=25 \mathrm{~Hz}$.

$h_{\text {wave }}<0.15 \delta$ is observed, and a layer of constant turbulent momentum flux $\tau_{\text {turb }}(\eta)=u_{*}^{2}$, in which the velocity depends on the vertical coordinate logarithmically, occurs within the layer of constant momentum flux.

As a rule, in wind tunnels and wind and wave channels, the thickness of the constant-flux layer is very small; for example, in these experiments, it was about $3 \mathrm{~cm}$. The measurement of the wind speed and temperature at such a small distance from the surface of a waved water surface, especially at strong winds, is a difficult problem, mainly due to the effect of spray torn off the wave crests. However, the parameters of the constant-flux layer can be obtained from measurements in the "wake" part of the turbulent boundary layer, as the profiles of velocity defects in near-wall turbulent flows are self-similar:

$$
\frac{U_{\max }-U(z)}{u_{*}}=F\left(\frac{z}{\delta}\right),
$$

where $U_{\max }$ is the maximum speed in a turbulent boundary layer. According to Hinze (1959), for a nongradient turbulent boundary layer at a flat plate or in a tube, the following approximation of the self-similar velocity profile can be made: 


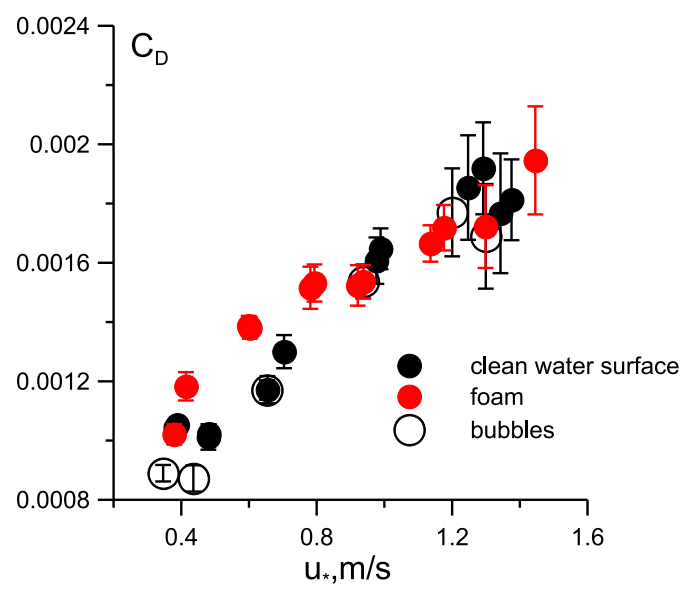

(a)

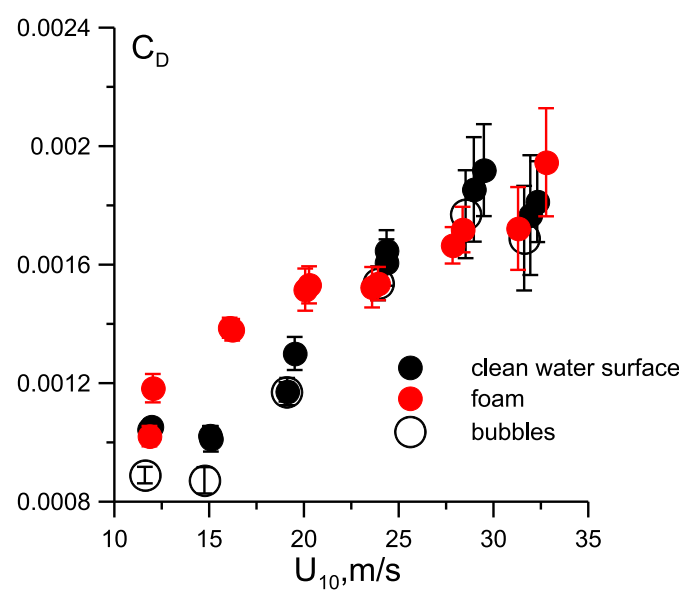

(b)

FIG. 3. Surface drag coefficient vs (a) friction velocity and (b) 10-m wind speed. Black symbols indicate a clean water surface; red symbols indicate the presence of SLS foam; open circles indicate the presence of bubbles produced by the foam generator without SLS.

$$
U_{\max }-U(z)=\left\{\begin{array}{lc}
u_{*}\left[-\frac{1}{\kappa} \ln (z / \delta)+\alpha\right] ; & z / \delta<0.15 \\
\beta u_{*}(1-z / \delta)^{2} ; & z / \delta>0.15
\end{array}\right.
$$

where $\kappa=0.4$ is the von Kármán constant.

The constants $\alpha$ and $\beta$ can be obtained from the best fitting of the experimental data. According to Hinze (1959), the values of the constants are $\alpha=1$ and $\beta=$ 9.6 for a nongradient turbulent boundary layer and $\alpha=1$ and $\beta=7.1$ for turbulent Poiseuille flow in a pipe. The experiments in the wind-wave flume show that the profile of the velocity defect in the airflow above the waved water surface is also self-similar and that the velocity profile can be approximated by Eq. (3) (see Troitskaya et al. 2012). In these experiments, $\alpha=1.5$ and $\beta=9.12$ (see Kandaurov et al. 2014).

Given the self-similarity of the airflow velocity profile in the channel, the parameters of the logarithmic boundary layer can be obtained from the measurements in the wake part of the turbulent boundary layer. First, the parameters of the turbulent boundary layer $\left(U_{\max }\right.$, $\beta u_{*}$, and $\delta$ ) are retrieved from the best fit of the experimental data with Eq. (3) for $z / \delta>0.15$. Second, the parameters of the logarithmic boundary layer are calculated with Eq. (1) for $z / \delta<0.15$ where

$$
z_{0}=\delta \exp \left(-\kappa U_{\max } / u_{*}+\alpha \kappa\right)
$$

The drag coefficient readily follows from Eqs. (1) and (4)

$$
C_{D}=\frac{\kappa^{2}}{\left[\kappa U_{\max } / u_{*}-\alpha \kappa+\ln \left(H_{10} / \delta\right)\right]^{2}} .
$$

Figure 3 presents the dependencies of the surface drag coefficient on friction velocity and $10-\mathrm{m}$ wind speed. At wind friction velocity speeds below $0.9 \mathrm{~m} \mathrm{~s}^{-1}\left(U_{10} \approx\right.$ $\left.25 \mathrm{~m} \mathrm{~s}^{-1}\right)$, the presence of foam increases the drag coefficient, while at higher wind speeds, no statistically significant dependence of the drag coefficient on the presence of foam is observed. One can also see that local production of short-lived bubbles by the foam generator without the introduction of SLS solution does lead to significantly different results to the case of clean water.

\section{b. Surface roughness}

In this experiment, the surface roughness is caused by surface waves and foam, created either artificially by the foam generator or naturally through wave breaking.

\section{1) SURFACE WAVE PARAMETERS}

The wind-wave field parameters in the flume were measured by three wire gauges positioned at the apices of a $2.5-\mathrm{cm}$-sided equilateral triangle. The data sampling rate was $100 \mathrm{~Hz}$. Three-dimensional space-time spectra were obtained from the measured data with the Fourier Directional Method (FDM) algorithm (see details in Troitskaya et al. 2012), similar to the Wavelet Directional Method (WDM) suggested by Donelan et al. (1996). This data processing yields the 3D spectra of surface waves $S(\omega, k, \theta)$ depending on wave 


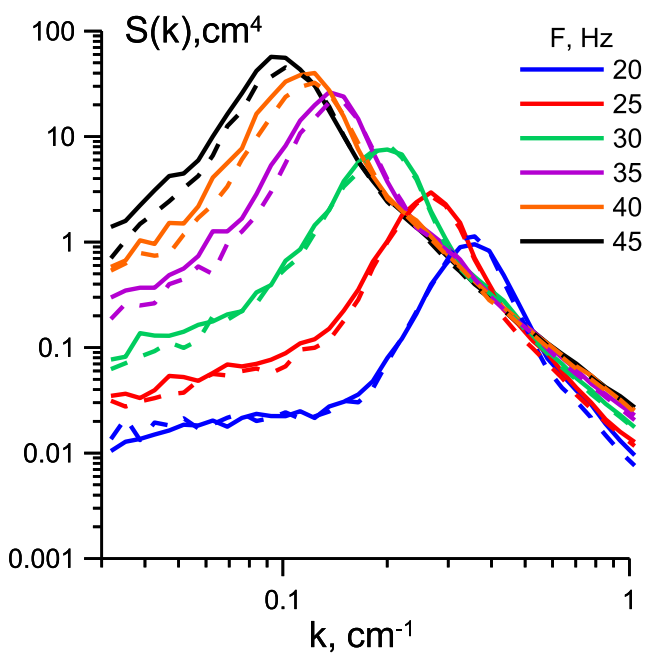

(a)

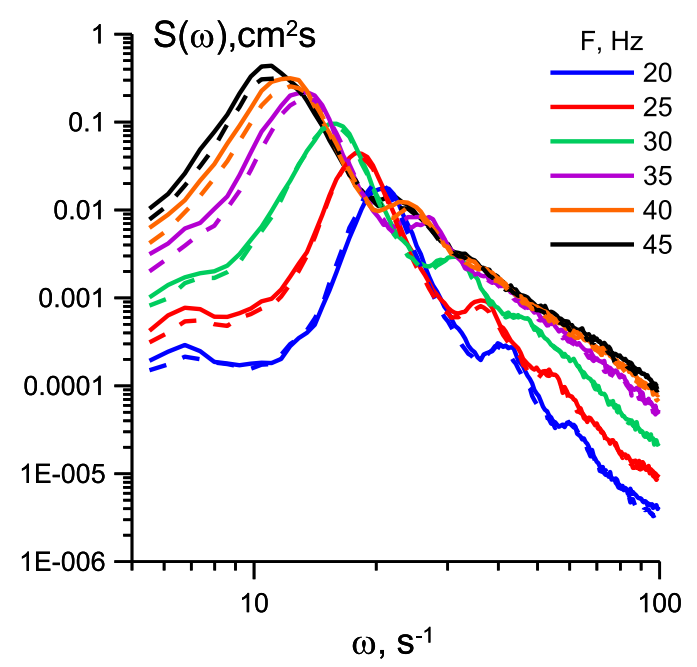

(c)

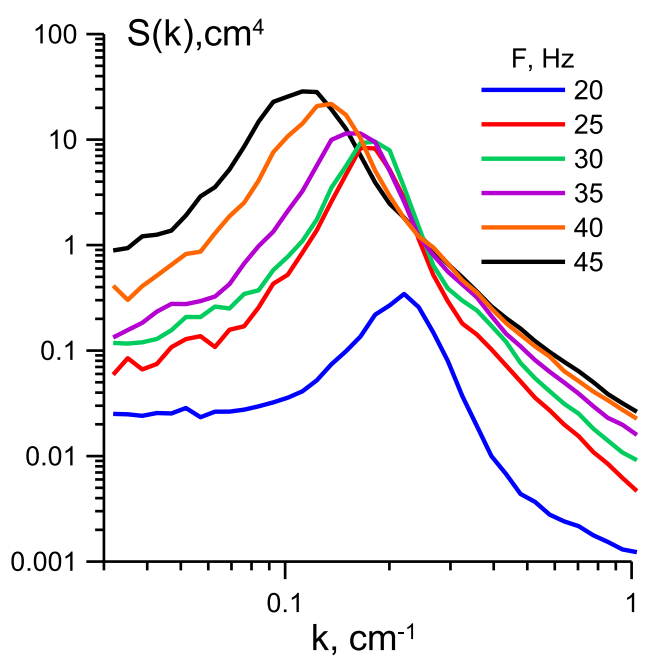

(b)

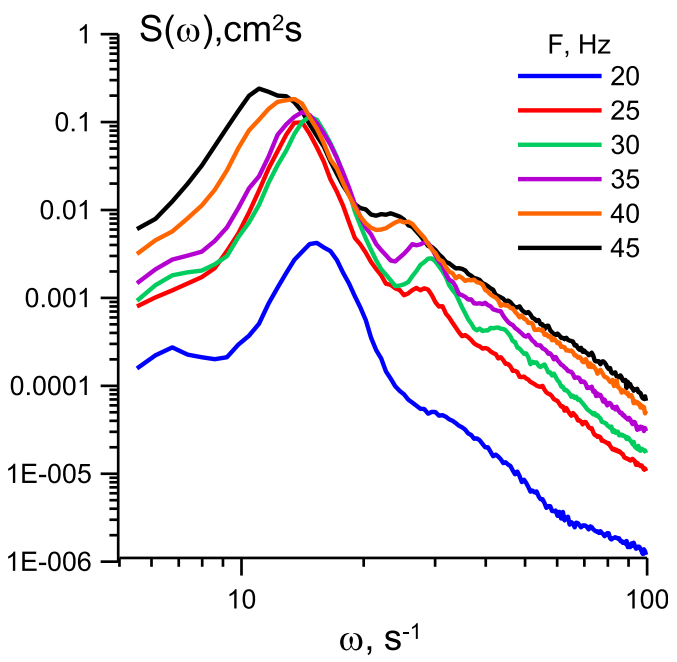

(d)

FIG. 4. Omnidirectional wavenumber and frequency spectra-derived profiles for six fan rotation frequencies: 20 (blue), 25 (red), 30 (green), 35 (purple), 40 (orange), and $45 \mathrm{~Hz}$ (black) in different regimes: (a),(c) clean water (solid curves) and foam generation in clean water and (b),(d) foam generation with SLS.

frequency $\omega$, wavenumber $k$, and the angle with respect to wind direction $\theta$. Integrating the spectra obtained $S(\omega, k, \theta)$ with the wavenumber or frequency gives temporary $S(\omega, \theta)$ or spatial $S(k, \theta)$ angular spectra, respectively. Integrating with $\theta$ gives the omnidirectional frequency and wavenumber spectra. The upper limit of the wavenumber spectrum is prescribed by the distance between the wave staffs $d, k_{u}=\pi / d$; in the configuration used, $k_{u}=1.25 \mathrm{~cm}^{-1}$. The omnidirectional wavenumber and frequency spectra obtained in different regimes are shown in Fig. 4.

It is clear that the presence of heavy foam modifies the surface wave spectra. The most striking effect is suppression of the downshift in the peak frequency $\omega_{p}$ and wavenumber $k_{p}$, with an increase of the wind velocity for $u_{*}<0.8 \mathrm{~m} \mathrm{~s}^{-1}$. This effect is shown explicitly in Figs. 5a and 5b, where the dependencies of $\omega_{p}$ and $k_{p}$ on wind friction velocity are presented for different regimes. The significant wave height is only slightly suppressed in the presence of artificial foam (Fig. 6a), but, in combination with a downshift in the peak wavenumber, it causes a strong decrease in the MSS (Fig. 6b). Consequently, we can expect a decrease in the nonlinearity of the surface wave field and a reduction in form drag when foam is present at the water surface. Analysis of the 2D wavenumber-angular surface wave 


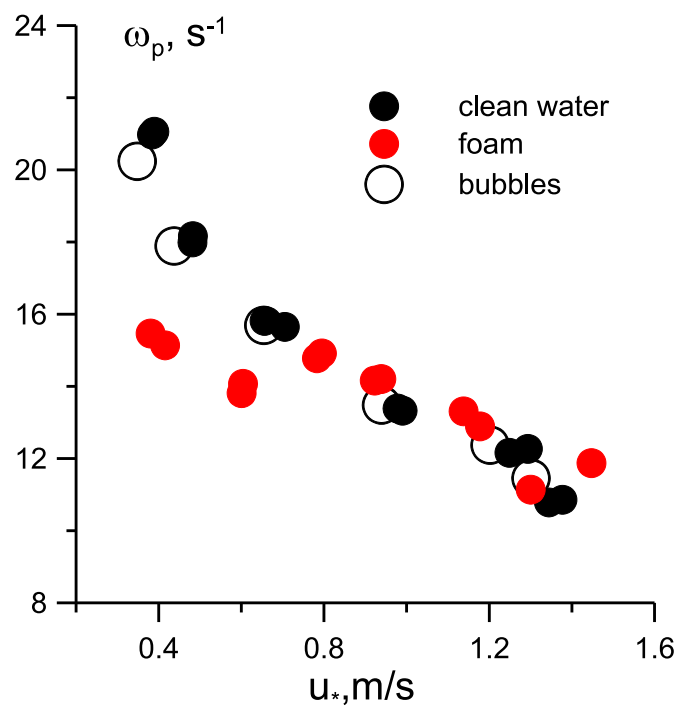

(a)

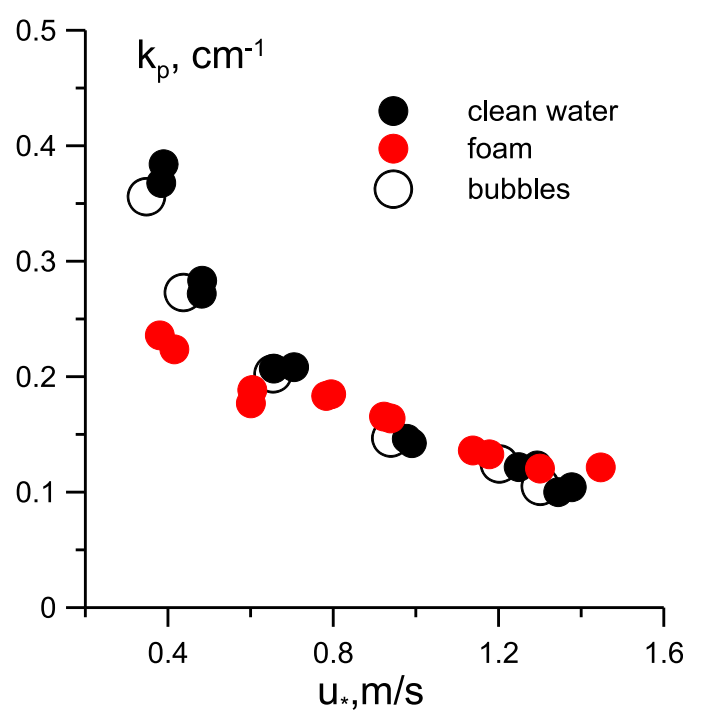

(b)

FIG. 5. Dependence of (a) $\omega_{p}$ and (b) $k_{p}$ on wind friction velocity under different regimes. Black symbols indicate a clean water surface, red symbols indicate the presence of SLS foam, and open circles indicate the presence of bubbles produced by the foam generator without SLS.

spectra retrieved by the FDM algorithm from the three-channel array measurements of the water surface elevation shows a reduction in the widths of the angular spectra where foam is present (see Fig. 7a). The effect is more pronounced at lower winds (Fig. 7b). This is most likely a consequence of reduced nonlinearity of the wave field. Taking this into account, we could expect a reduction of the form drag of the water surface in the presence of foam compared to with clean water. However, comparison with Fig. 3 shows that the surface drag coefficient instead increases when foam is present at the water surface. This means that foam produces additional roughness. To model this effect, we need to determine the parameters

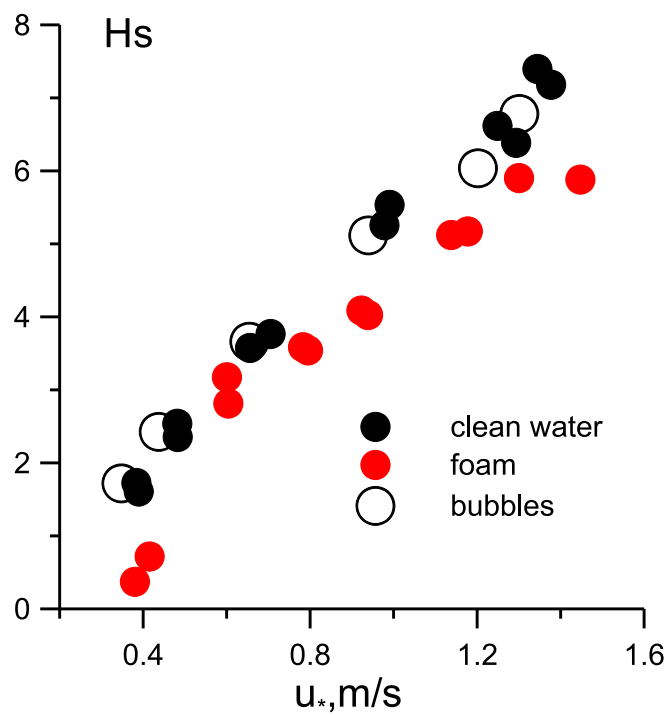

(a)

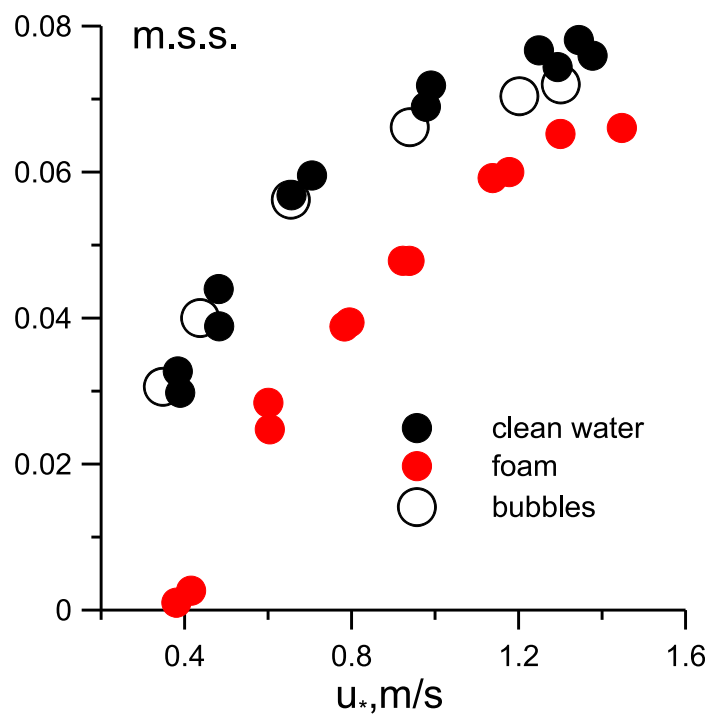

(b)

FIG. 6. Dependence of (a) significant wave height and (b) mean square slope on wind friction velocity under different regimes. Black symbols indicate a clean water surface, red symbols indicate the presence of SLS foam, and open circles indicate the presence of bubbles produced by the foam generator without SLS. 


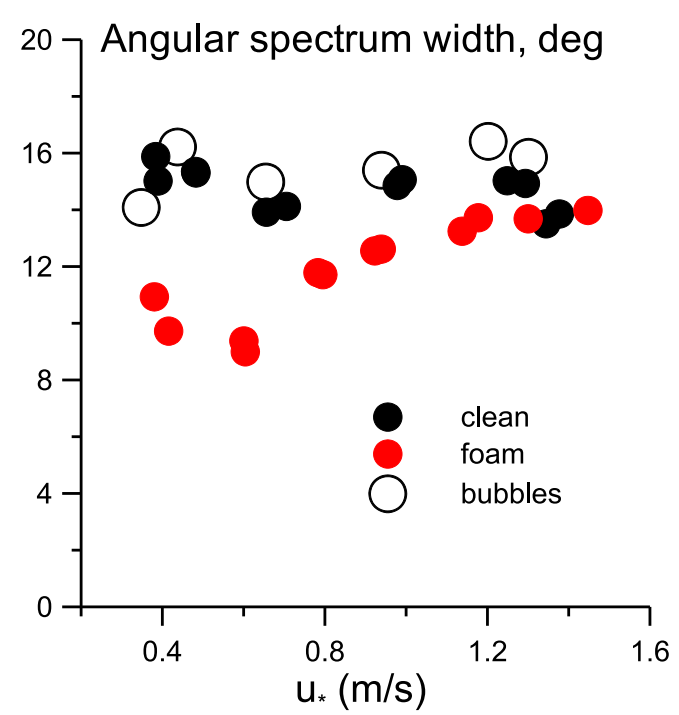

(a)
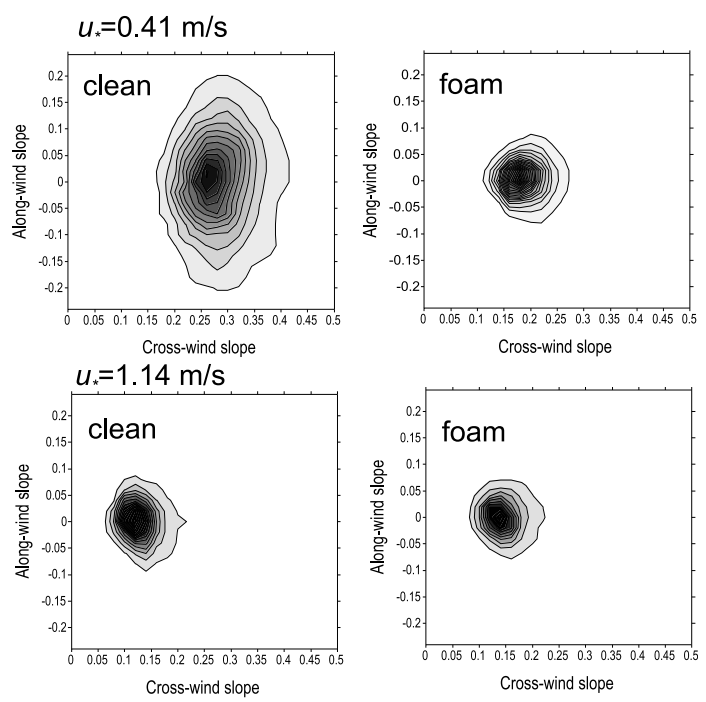

(b)

FIG. 7. (a) Dependence of angular spectrum width on wind friction velocity under different regimes. Black symbols indicate a clean water surface, red symbols indicate the presence of SLS foam, and open circles indicate the presence of bubbles produced by the foam generator without SLS. (b) Examples of angular spectra: (top) $u_{*}=0.41$ and (bottom) $u_{*}=1.14 \mathrm{~m} \mathrm{~s}^{-1}$; (left) foam-free water surface and (right) foam-covered water surface.

of the foam present at the water surface in these experiments.

\section{2) FOAM PARAMETERS}

The foam parameters were studied with the use of the optical shadow method, implemented through top-view video capture and underwater illumination. The video was taken through the transparent top wall of channel section 7 (6.5-m fetch). The camera was mounted vertically at a distance of $43 \mathrm{~cm}$ from the water surface. The record rate was 12 frames per second (fps) with a resolution of $113 \mu \mathrm{m}$ per pixel. A horizontal diffuser screen was installed under the working section at a depth of $370 \mathrm{~mm}$. The screen was illuminated from below by an array of $25(5 \times 5)$ white waterproof 10-W LED light sources.

The proportion of the surface area covered by foam was calculated on the basis of the number of pixels that were darker than a certain threshold. To eliminate the influence of noise and small optical inhomogeneities, the original images were divided into regions, in each of which the average brightness was calculated, that is, the resolution of the frames was decreased 50 times on each side. A single background image was calculated as the median value for each pixel based on recordings taken with no wind. The background image was subtracted from each frame, and then a common histogram (brightness distribution of areas in the image) was constructed for all frames in the record. For each histogram, it is possible to calculate the proportion of regions with a brightness below the threshold, which naturally depends on the choice of the threshold. The threshold was determined through direct calculation of the foam-covered area using morphological imaging. This was conducted in several stages: the background was subtracted from the original image and the result was cropped; the image was binarized with a fixed threshold; morphological opening was performed with the structural element of a "disk" of 1 pixel followed by morphological closure with a "disk" of 21 pixels; closed regions of the image were filled using a flood-fill operation. The ratio of the number of pixels found to the total number of pixels was taken as the fraction of the surface area covered with foam.

The foam coverage fraction of the water surface and the bubble size distribution were obtained on the basis of the results of processing the video data. At a constant rate of ejection of SLS solution from the foam generator, the area of the water surface covered by foam depended on the wind speed (Fig. 8a). At wind friction velocities below $0.8-0.9 \mathrm{~m} \mathrm{~s}^{-1}$ (10-m wind speed below $22-25 \mathrm{~m} \mathrm{~s}^{-1}$ ), the fractional foam coverage decreased with increasing wind speed, and concentration of foam near the wave crests was observed. At wind friction velocities exceeding $0.8-0.9 \mathrm{~m} \mathrm{~s}^{-1}$, intense wave breaking was observed, accompanied by 


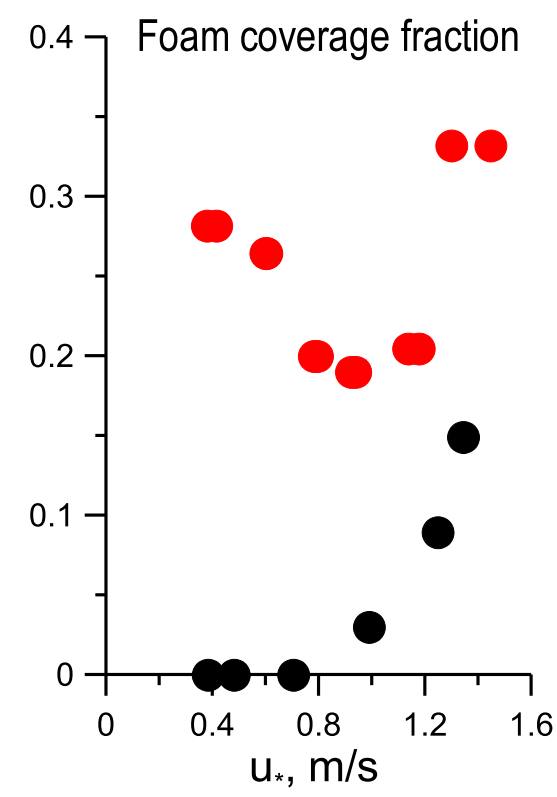

(a)

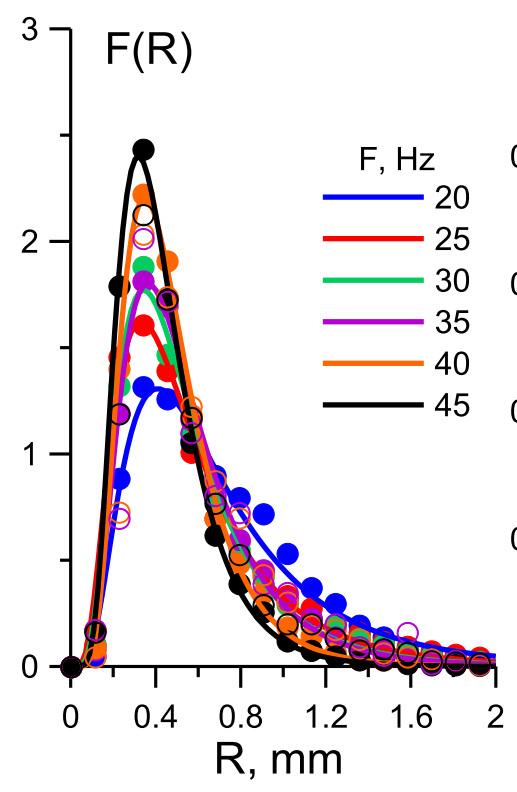

(b)

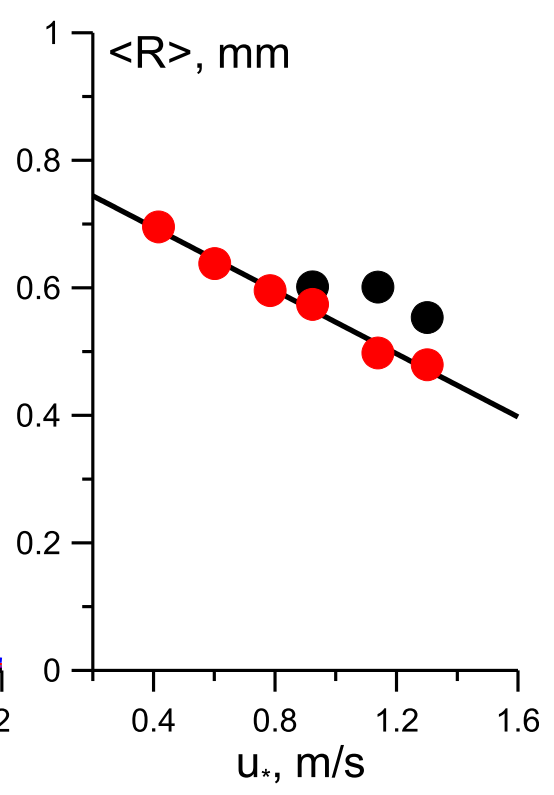

(c)

FIG. 8. (a) Fractional foam coverage vs wind friction velocity (red points, artificial foam; black points, natural foam from wave breaking). (b) Size distributions of bubbles for different airflow velocities for the cases of artificial (closed symbols) and natural (open symbols) foam and approximations by the lognormal function (line); colors denote different fan rotation frequencies: 20 (blue), 25 (red), 30 (green), 35 (purple), 40 (orange), and $45 \mathrm{~Hz}$ (black). (c) Average foam bubble radius vs wind friction velocity [circles indicate the experimental data for the cases of artificial (red) and natural foam (black); line indicates the best fit of the data for artificial foam by Eq. (5)].

the formation of foam even when the foam generator was not operated (see Fig. 8a).

Images of the water surface when covered by foam enable us to measure the size distribution of the foam bubbles. From each record, several frames were selected on which the foam is clearly visible. The areas on which the bubbles merge (located in several layers) or that were unusable for automatic detection of the bubble size were painted over manually. For the areas remaining after this preliminary filtering, all circles were found through two-stage circular Hough transform, and their radius distribution was calculated. The size distribution of the foam bubbles is well approximated by a lognormal distribution (see Fig. 8b):

$$
F(R)=\frac{1}{\sqrt{2 \pi} \sigma R} \exp \left[-\frac{1}{2 \sigma^{2}} \ln ^{2}\left(\frac{R}{R_{0}}\right)\right] .
$$

The corresponding average bubble radius,

$$
\langle R\rangle=R_{0} \exp \left(\frac{\sigma^{2}}{2}\right),
$$

decreased with an increase in wind forcing (see Fig. 8c). For the case of artificial foam created by the foam generator, the dependence of $\langle R\rangle$ on the wind friction velocity was best fitted by a linear function:

$$
\langle R\rangle=R_{0}-R_{1} u_{*},
$$

where the estimates of the average and $95 \%$ confidence interval for the regression coefficients are $R_{0}=$ $0.79 \pm 0.02 \mathrm{~mm}$ and $R_{1}=0.25 \pm 0.02 \mathrm{~mm} \mathrm{~m}^{-1} \mathrm{~s}$. The average radii of the foam bubbles in the case of natural foam arising due to wave breaking were about $10 \%$ higher than for the artificial foam (see Fig. 8c), presumably due to prevention of bubble coalescence in the presence of small quantities of SLS in the artificial foam.

Similarly to Troitskaya et al. (2012), we plot $C_{D}$ versus wave MSS in Fig. 9a. For both clean water and water covered with artificially produced foam, the dependencies of $C_{D}$ on MSS appear to be close to linear functions, with the slope differing depending on the fraction of foam coverage. For our experimental data, we found a double linear regression between the drag coefficient $C_{D}$, MSS and fraction of foam coverage $s_{\text {foam }}$ :

$$
C_{D}=10^{-3}\left(C_{0}+C_{1} \mathrm{MSS}+C_{2} S_{\text {foam }}\right),
$$




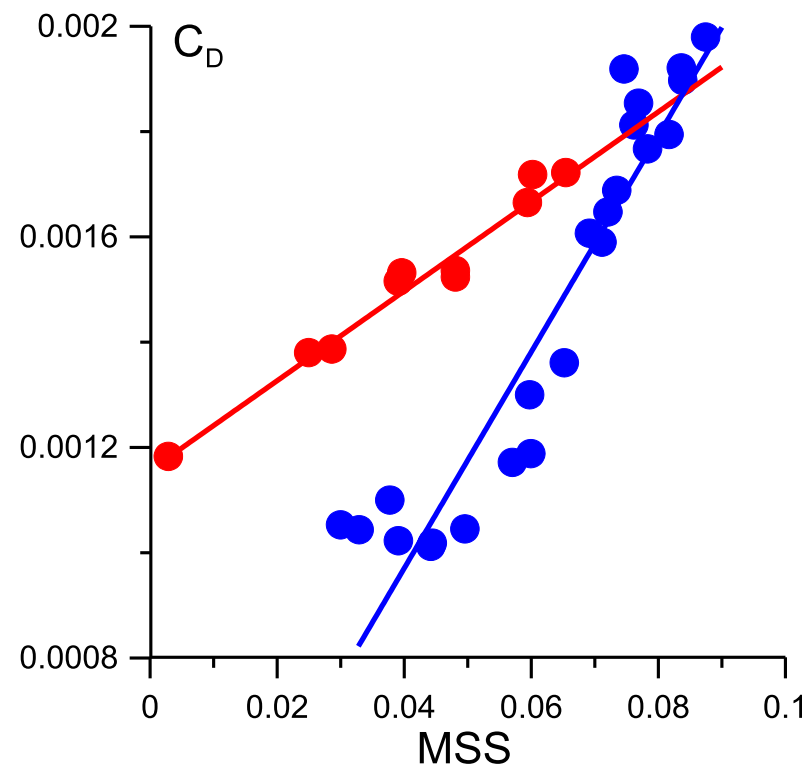

FIG. 9. Surface drag coefficient $C_{D}$ vs mean square slope. Blue symbols indicate clean water, and red symbols indicate water covered with foam.

where the estimates of the average and $95 \%$ confidence interval for the regression coefficients are $C_{0}=0.552 \pm$ $0.12, C_{1}=13.43 \pm 1.70$, and $C_{2}=1.71 \pm 0.34$. The coefficient of determination of the model equation [Eq. (6)] is 0.9 .

The linear regression model [Eq. (6)] shows that the surface drag coefficient increases both with an increase in the MSS of the water surface and with the fractional foam coverage, that is, the presence of foam results in an increase in the aerodynamic resistance of the water surface. However, we should take into account that the MSS of the water surface and the fractional foam coverage are not independent variables: the statistical analysis showed the presence of a negative correlation between the variables MSS and $S_{\text {foam }}$ with a correlation coefficient of -0.252 . This is confirmed by Fig. 6b, which shows a decrease in MSS if foam is present at a given level of wind forcing. To quantify the dependence of MSS on the fractional foam coverage at a specific wind speed, we plotted the ratio $\operatorname{MSS}\left(u_{*}, S_{\text {foam } M}\right) / \operatorname{MSS}\left(u_{*}, S_{\text {foam } N}\right)$ against the difference $S_{\text {foam } M}-S_{\text {foam } N}$ for each pair of MSS, $\operatorname{MSS}\left(u_{*}, S_{\text {foam } M}\right)$ and $\operatorname{MSS}\left(u_{*}, S_{\text {foam } N}\right)$, measured at a certain wind speed $u_{*}$ but at different values of fractional foam coverage $S_{\text {foam } M}$ and $S_{\text {foam } N}$ (with and without artificially produced foam; see Fig. 10). The close grouping of points on the dependence between $\operatorname{MSS}\left(u_{*}, S_{\text {foam } M}\right) / \operatorname{MSS}\left(u_{*}, S_{\text {foam } N}\right)$ and $S_{\text {foam } M}-S_{\text {foam } N}$ in Fig. 10 indicates the possibility of factorization of the function $\operatorname{MSS}\left(u_{*}, S_{\text {foam }}\right)$ and the exponential dependence of MSS on fractional foam

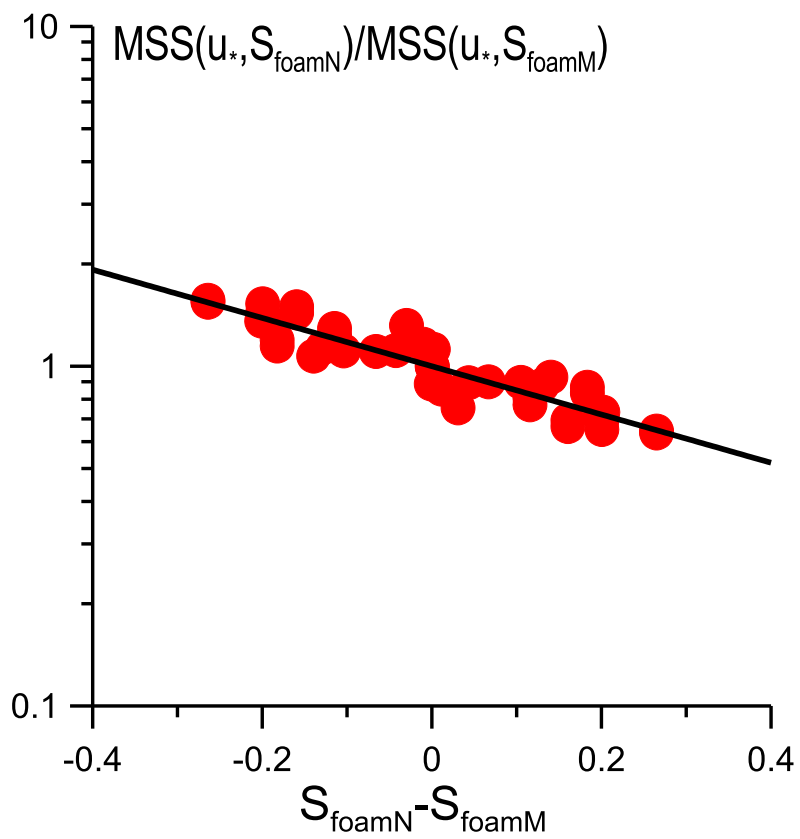

FIG. 10. Approximation of the dependence of MSS on friction velocity $u *$ and fractional foam coverage $S_{\text {foam }}$.

coverage. The exponential best fit of the experimental points in Fig. 10 yields

$$
\begin{aligned}
\operatorname{MSS}\left(u_{*}, S_{\text {foam }}\right) & =\operatorname{MSS}_{0}\left(u_{*}\right) C\left(S_{\text {foam }}\right) \\
C\left(S_{\text {foam }}\right) & =\exp \left(-\beta S_{\text {foam }}\right),
\end{aligned}
$$

where the estimates of the average and $95 \%$ confidence interval for $\beta$ are 1.64 and 0.20 , respectively.

\section{Comparison of the prediction of the drag coefficient within the quasi-linear model with the experimental results}

The resulting measurements were compared with the predictions of a quasi-linear model of the atmospheric boundary layer over a waved water surface described by Troitskaya et al. (2012, 2014). The model is based on RANS (Reynolds-averaged Navier-Stokes) equations closed with the Boussinesq hypothesis. To avoid strong geometric nonlinearity, the RANS equations are formulated in wave-following curvilinear coordinates $\left(\xi_{1}, \xi_{2}, \eta\right)$. The model describes the wind-wave interaction in the quasi-linear approximation, when the waveinduced disturbances of the atmospheric turbulent boundary layer are described in the linear approximation and the momentum flux from wind to waves is the only nonlinear effect in the model. The latter describes the wave form drag. The quasi-linear model assumes no separation from the crests of the surface waves of the 
wind flow averaged over turbulent fluctuations. The applicability of this assumption is justified by the laboratory experiments by Troitskaya et al. (2011) and Buckley and Veron (2016) and the direct numerical simulation by Yang and Shen (2010) and Druzhinin et al. (2012). Note, that this approach alters the model by Kudryavtsev and Makin (2007), where the airflow separation from the wave crests is assumed as essential factor. The input parameters of the model are the friction velocity in the turbulent boundary layer and the 3D wavenumber-frequency angular surface wave spectrum. The output parameters are the $10-\mathrm{m}$ wind speed and the drag coefficient of the water surface. The input and output parameters are measured independently and can be used for verification of the model.

The main difference between the present model and that developed by Troitskaya et al. $(2012,2014)$ is the parameterization of the eddy viscosity coefficient. The previous model exploited the self-similar eddy viscosity $\nu$ for the turbulent boundary layer:

$$
\nu=\nu_{a} f\left(\frac{u_{*} \eta}{\nu_{a}}\right)
$$

where $\nu_{a}$ is the molecular viscosity of air and $\eta$ is the wave-following curvilinear coordinate transverse to the water surface.

Troitskaya et al. $(2012,2014)$ used the approximation for $f$ that Smol'yakov (1973) obtained in laboratory experiments of a turbulent boundary layer over an aerodynamically smooth plate:

$$
\nu=\nu_{a}\left\{1+\kappa \frac{u_{*} \eta}{\nu_{a}}\left[1-e^{-(1 / L)\left(u_{*} \eta / \nu_{a}\right)^{2}}\right]\right\},
$$

where $L=22.4$ for an aerodynamically smooth surface.

In the present paper, we use the model eddy viscosity coefficient, which takes into account the foam at the water surface. We use a more simple equation for $\nu$ :

$$
\nu=\kappa u_{*}\left(\eta+z_{00}\right)
$$

where $\kappa=0.4$ is the von Kármán constant and the parameter $z_{00}$ is the effective roughness height. Below, we will use the term "skin roughness height" to distinguish $z_{00}$ from the common roughness height of the sea surface under wind forcing, $z_{0}$, which is also determined by the form drag of surface waves. To illustrate this difference directly, consider the gradient approximation for the turbulent momentum flux in Eq. (2) with the eddy viscosity parameterized by Eq. (9):

$$
\kappa u_{*}\left(\eta+z_{00}\right) \frac{d U}{d \eta}+\tau_{\text {wave }}(\eta)=u_{*}^{2} .
$$

Parameter $\tau_{\text {wave }}(\eta)$ is calculated below within the quasilinear model.

Integrating Eq. (10) gives the average velocity profile in the turbulent boundary layer for $\eta \gg h_{\text {wave }}$ :

$$
U(\eta)=\frac{u_{*}}{\kappa} \ln \frac{\eta}{z_{00}}-\int_{0}^{\infty} \frac{\tau_{\text {wave }}(z) d z}{\kappa u_{*}\left(z+z_{00}\right)} .
$$

The second term on the right-hand side of Eq. (11) is the contribution of the form drag of the water surface to the wind velocity profile. Obviously, it follows from Eq. (11) that the roughness height of the water surface $z_{0}$ in Eq. (1), relates to $z_{00}$ as follows:

$$
z_{0}=z_{00} \exp \left[\int_{0}^{\infty} \frac{\tau_{\text {wave }}(z) d z}{u_{*}^{2}\left(z+z_{00}\right)}\right]
$$

Notice a significant difference between this model and the similar model developed by Golbraikh and Shtemler (2016), who suggested that the common roughness height $z_{0}$ of a waved water surface partially covered by foam be parameterized by using the Charnock formula to obtain the roughness height of the foam-free fractions of the surface.

Golbraikh and Shtemler (2016) assumed that the roughness height of the surface can be represented as the superposition of the contributions of two types of underlying surface: 1) areas covered by foam and 2) clean surfaces. Here we make a similar assumption for the skin roughness height $z_{00}$. For the first type of surface, the skin roughness height is taken to be equal to $\alpha\langle R\rangle$, where $\langle R\rangle$ is the average foam bubble radius and $\alpha$ is the ratio of the aerodynamic to geometrical roughness of the foam. For solid roughness elements at a solid plain surface, the ratio of the aerodynamic to geometric roughness heights is about $1 / 30$ according to Bagnold (1941). For foam at a water surface (white caps and streaks) at strong and hurricane winds, Golbraikh and Shtemler (2016) and Shtemler et al. (2010) notice that this ratio is of the order of unity. This discrepancy with the results by Bagnold (1941) can be explained by the influence of two factors: the form drag of the surface waves and the difference in elasticity of the roughness elements. Below, we estimate the parameter $\alpha$ from the measurements made in this study.

Finally, the eddy viscosity coefficient in the turbulent boundary layer over of a smooth surface partially covered by foam is

$$
\nu=\kappa u_{*}\left[\eta+z_{00 \text { free }}\left(1-s_{\text {foam }}\right)+\alpha\langle R\rangle S_{\text {foam }}\right],
$$




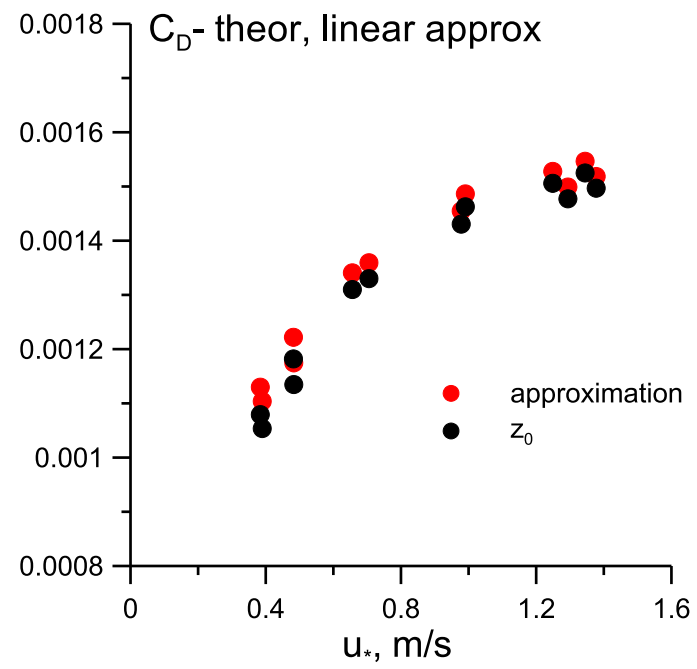

(a)

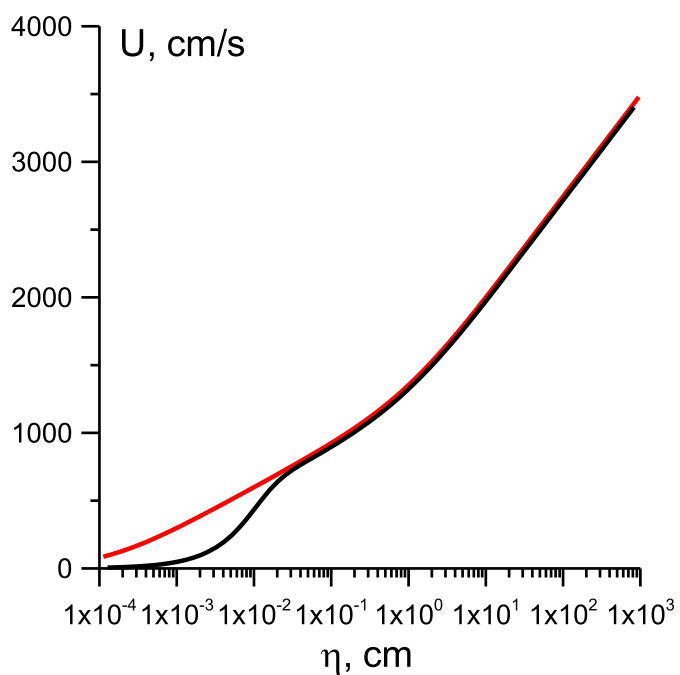

(b)

FIG. 11. Verification of the applicability of the simplified model of eddy viscosity for description of the turbulent boundary layer above a smooth wavy surface. (a) Drag coefficient vs friction velocity: red symbols are calculations with the eddy viscosity given by Eq. (8), and black symbols are calculations with the eddy viscosity given by Eq. (13). (b) Examples of airflow velocity profiles over a smooth surface calculated within the two models of eddy viscosity: black curve is Eq. (8), and red curve is Eq. (13).

Here, $s_{\text {foam }}$ is the fraction of the water area covered by foam and $z_{00 f r e e}$ is the roughness height of the aerodynamically smooth surface:

$$
z_{00 \mathrm{free}}=\frac{0.11 \nu_{a}}{u_{*}} .
$$

To determine the complete drag coefficient, we have to calculate the form drag of the waves. In the absence of foam at the water surface, $S_{\text {foam }}=0$, the eddy viscosity in the turbulent boundary layer over a smooth surface is described by Eq. (13). It should be noted that this is a fairly simple approximate model that strongly diverges from the more sophisticated and accurate model of Smol'yakov (1973) in the viscous and transitional sublayers, although the two models practically coincide in the logarithmic part of the turbulent boundary layer. We first investigated the applicability of the simplified model, Eq. (13), to describing the form drag of an aerodynamically smooth waved water surface through the quasi-linear model of Troitskaya et al. (2012, 2014). Figure 11a shows the calculated surface drag coefficient in aerodynamically smooth flow under Smol'yakov's model, Eq. (8), and the simple model, Eq. (13), with $S_{\text {foam }}=0$. Input surface wave spectra were taken from experiments without artificial foam at the water surface. It is obvious from Fig. 11a that, for this experiment, the simplified model of the eddy viscosity gives values for the surface drag coefficient that are very close to the results of the more accurate model.

The examples in Fig. 11b show that the average airflow velocity profiles generated by the two models are distinguishable only in the viscous sublayer and transitional zone, at a distance of less than $1 \mathrm{~mm}$ from the surface, while the wind-wave momentum exchange occurs mostly in the logarithmic layer. From the physical point of view, this is due to the fact that the form drag is determined by the aerodynamic pressure, which has a vertical scale comparable with a wavelength far exceeding the scales of the viscous sublayer and the transitional zone.

The spectra of surface waves, the fractional foam coverage $s_{\text {foam }}$, and the average sizes of bubbles $\langle R\rangle$ measured at certain values of wind friction velocity (see Figs. 8a,b) were used as the input parameters for the quasi-linear model using the eddy viscosity coefficient given by Eq. (13). The fitting coefficient $\alpha=$ 0.37 was obtained from the best fit of one of the series of experiments (in the case of artificial foam at the water surface). The same $\alpha$ was then used in calculations within the quasi-linear model for other experimental runs. Comparing the experiments in Figs. 12a-c shows that the quasi-linear model using the eddy viscosity coefficient in Eq. (13) reproduces the aerodynamic resistance of the water surface well. In this model, the surface drag coefficient is determined on the basis of the form drag of waves, as calculated within 


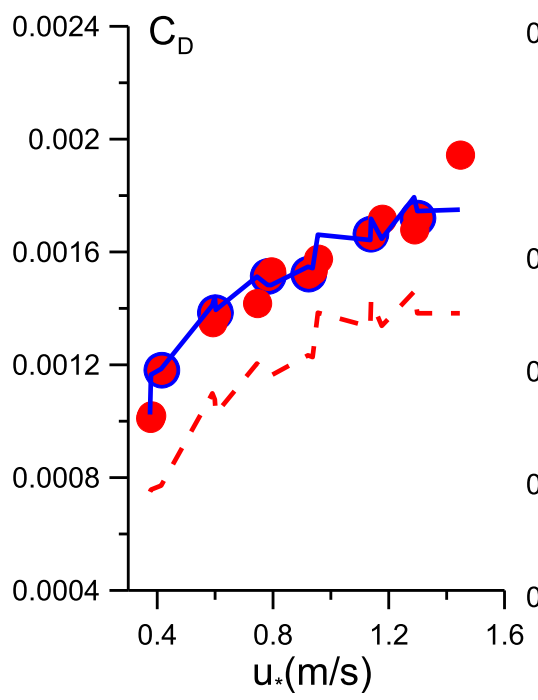

(a)

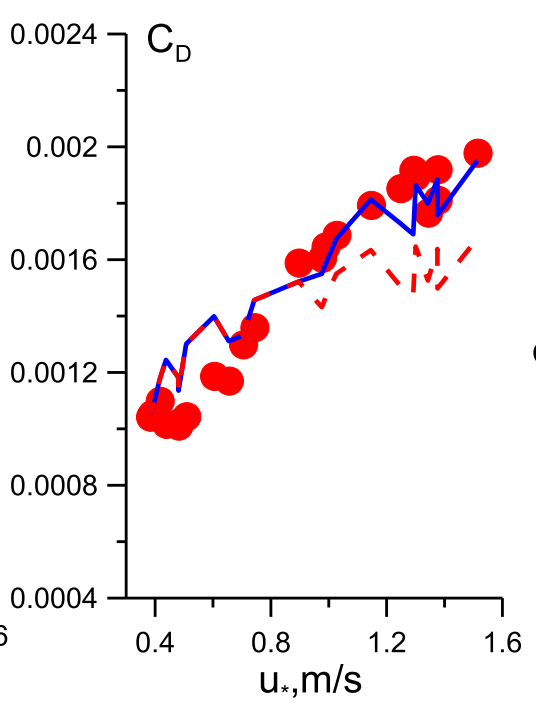

(b)

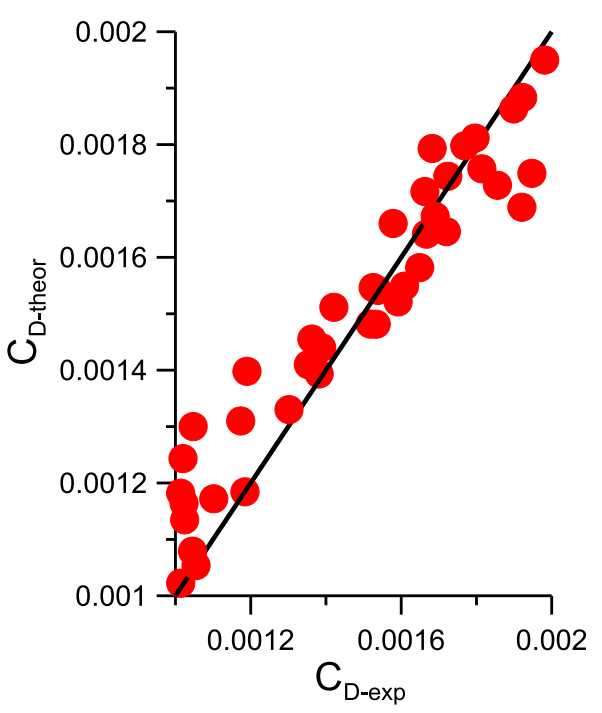

(c)

FIG. 12. Drag coefficient vs friction velocity of the airflow for the cases of (a) artificial foam at the water surface and (b) clean water. Red circles are experimental data (circles with blue rims were used for retrieving the fitting coefficient $\alpha$ ); blue solid line indicates calculations within the quasi-linear model with the eddy viscosity coefficient given by Eq. (13); and dashed line indicates calculations within the quasi-linear model using the eddy viscosity coefficient for a smooth surface. (c) Theoretical predictions against experimental data.

the quasi-linear approximation for the measured surface wave spectra, and the aerodynamic roughness of a water surface partially covered by foam. Note that the coefficient $\alpha$, the ratio of the skin roughness height of the foam-covered surface and the sizes of the roughness elements, is of the order of unity, similarly to the estimate of the ratio of aerodynamic and geometrical roughness heights of a waved foamcovered water surface arrived at by Golbraikh and Shtemler (2016). Figure 12b also shows that, in the case of clean water, taking into account foam coverage due to wave breaking improves prediction of the drag coefficient at wind speeds above $25 \mathrm{~m} \mathrm{~s}^{-1}$ noticeably compared with the smooth surface model. Note that comparing Fig. 12a and Fig. 12b enables us to conclude that the quasi-linear model is in better agreement with the data collected for cases where there is artificial foam coverage. It seems this can be explained by the lower steepness of the wave field, which should lead to fewer nonlinear effects than in cases involving clean water.

\section{Estimates of the effect of the surface drag coefficient of the water surface under field conditions}

Using the experimental data described above, we estimated the effect of foam at the water surface on its aerodynamic resistance at high winds. The main influences here are the change in the roughness height of the water surface caused by the presence of foam bubbles and the decrease in the MSS of the waved water surface at fixed wind forcing due to enhanced wave dissipation when foam is present at the water surface. In this section, we apply the results obtained in the laboratory environment to estimate the surface drag coefficient of a water surface covered by foam under field conditions. Obviously, due to the major differences between field and laboratory conditions, the preliminary estimates described here can only be considered as a demonstration of qualitative effects.

For these estimates, we used the Troitskaya et al. (2012, 2014) quasi-linear model described in section 4. As inputs, we used the spectrum of Donelan et al. (1985) for the dominant waves and the saturation spectrum of Phillips (1958) modified for the presence of foam in the spectral tail. This assumption is in agreement with Young (2017), who proved the applicability of the Donelan et al. (1985) spectra at hurricane wind speeds. The corresponding slope spectrum was as follows:

$$
F(k, \omega, \theta)=\frac{2}{\pi} B(k) k^{-1} \cos ^{2} \theta \delta[\omega-\omega(k)],
$$

with 


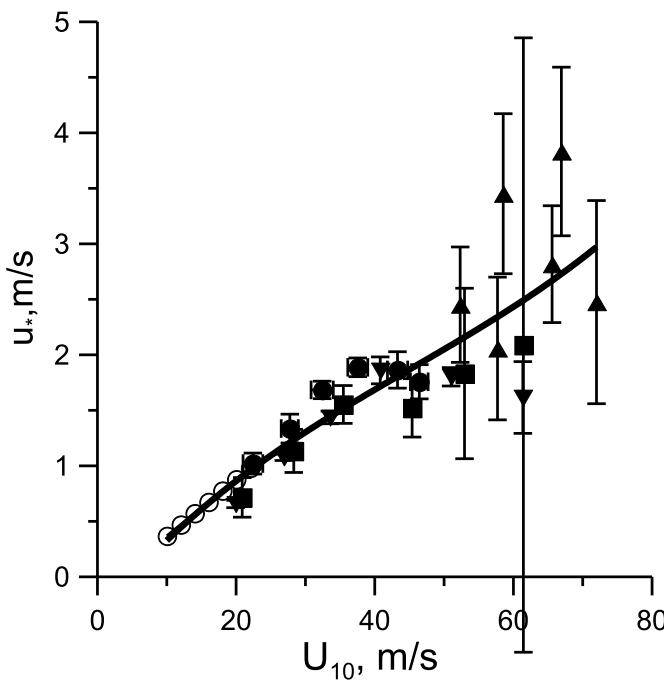

(a)

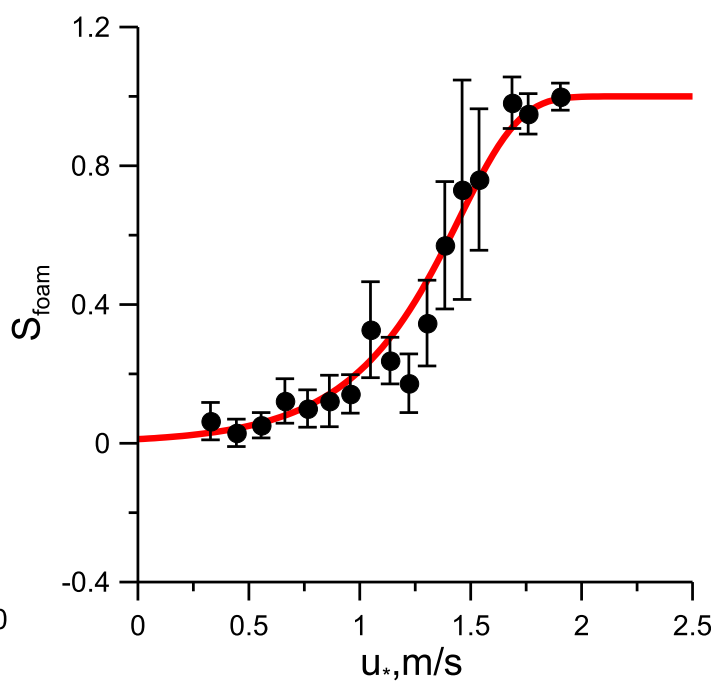

(b)

FIG. 13. (a) Empirical dependence $u_{*}\left(U_{10}\right)$ : squares indicate Richter et al. (2016), closed circles indicate Jarosz et al. (2007), downward-pointing triangles indicate Holthuijsen et al. (2012), upward-pointing triangles indicate Bell et al. (2012), and open circles indicate Eq. (16). Curve is the polynomial approximation, Eq. (17). (b) Experimental data on fractional foam coverage $S_{\text {foam }}$ from Holthuijsen et al. (2012) vs $u_{*}$ calculated by Eq. (17); the curve is the function Eq. (18).

$$
B_{l}=\frac{0.006}{2} \Omega^{0.55}\left\{\begin{array}{l}
\left(\frac{k}{k_{p}}\right)^{1 / 2} \exp \left[-\frac{5}{4}\left(\frac{k}{k_{p}}\right)^{2}[1.7+6 \log (\Omega)]\right. \\
C\left(S_{\text {foam }}\right)\left(\frac{k_{0}}{k_{p}}\right)^{1 / 2},
\end{array}\right.
$$

$$
\begin{aligned}
& \left.\exp \left((1 / 2)\left\{\left(\sqrt{k}-\sqrt{k_{p}}\right) \mid\left[0.08\left(1+4 \Omega^{-3}\right) \sqrt{k_{p}}\right]\right\}^{2}\right)\right], \quad k<k_{0} \\
& k>k_{0}
\end{aligned}
$$

Here, $\Omega=U_{10} \sqrt{k_{p}} / \sqrt{g}$ is the wave-age parameter, $k_{p}$ is the peak wavenumber of the wind-wave spectra, $k_{0}=$ $3 k_{p}$ is the cutoff wavenumber prescribing the upper limit of the energy-containing part of the spectrum as it is assumed in the model of the wind-wave spectrum, WAM (Komen et al. 1994). Parameter $C\left(S_{\text {foam }}\right)$ is a coefficient describing the suppression of waves in the presence of foam, which is prescribed by the fractional foam coverage $S_{\text {foam }}$. For the present estimates, its approximate value was taken from our laboratory results, Eq. (7). The dependence of the fractional foam coverage $S_{\text {foam }}$ on wind speed $U_{10}$ is given by Holthuijsen et al. (2012), retrieved from numerous areal images of the water surface. Notice that, according to Holthuijsen et al. (2012), the foam coverage of the water surface at high winds is the sum of whitecaps and streaks arising as a consequence of Langmuir circulation. Here, we express $S_{\text {foam }}$ via $u *$ using the $S_{\text {foam }}\left(U_{10}\right)$ dependencies in Holthuijsen et al. (2012).
To approximate the dependence $u_{*}\left(U_{10}\right)$, we used available field data on the resistance law of the ocean surface. For $U_{10}>20 \mathrm{~m} \mathrm{~s}^{-1}$, we used data on $u_{*}\left(U_{10}\right)$ from Richter et al. (2016) and Bell et al. (2012). The dependence $u_{*}\left(U_{10}\right)$ was also retrieved according to the definition $u_{*}=U_{10} \sqrt{C_{D}\left(U_{10}\right)}$ arrived at on the basis of data on $C_{D}\left(U_{10}\right)$ from Holthuijsen et al. (2012) and Jarosz et al. (2007). For $U_{10}<20 \mathrm{~m} \mathrm{~s}^{-1}$, we used the function $u_{*}\left(U_{10}\right)$ given by Foreman and Emeis (2010):

$$
u_{*}\left(U_{10}\right)=0.051 U_{10}-0.14 \mathrm{~m} \mathrm{~s}^{-1},
$$

where $u_{*}$ and $U_{10}$ are expressed in meters per second.

As can be seen below, the estimates of the surface drag coefficient are in agreement with this assumption.

The data are plotted together in Fig. 13a. The empirical function $u_{*}\left(U_{10}\right)$ was approximated by the cubic polynomial model 


$$
u_{*}\left(U_{10}\right)=v_{0}+v_{1} U_{10}+v_{2} U_{10}^{2}+v_{3} U_{10}^{3},
$$

where $v_{0}=-0.343, v_{1}=7.5 \times 10^{-2}, v_{2}=-8.6 \times 10^{-4}$, and $v_{3}=6.4 \times 10^{-6}$. The parameters of the model are statistically significant according to the Student's $t$ criterion.

Equation (17) was used to transform the experimental dependence $S_{\text {foam }}\left(U_{10}\right)$ from Holthuijsen et al. (2012) to the dependence $S_{\text {foam }}\left(u_{*}\right)$. The corresponding experimental points are shown in Fig. 13b. The experimental dependence was approximated by a function similar to that suggested by Holthuijsen et al. (2012):

$$
S_{\text {foam }}=a \tanh \left[b \exp \left(c u_{*}\right)\right] .
$$

In the approximation given by Eq. (18), a prior assumption was used that $a=1$. This corresponds to the assumption that the entire water surface is covered by foam at extremely high winds. With the use of the least squares method, the approximation gives $b=0.012 \pm$ 0.003 and $c=2.88 \pm 0.21$.

The eddy viscosity coefficient used in the quasi-linear model was defined by Eq. (13). The average radius of a foam bubble used in Eq. (13) was calculated on the basis of the empirical dependence, Eq. (5), obtained as a linear regression of the data for experiments with artificial SLS foam. These data are expected to correspond better to the case of oceanic saltwater than do data for natural foam in freshwater. This supposition is based on the suppression of bubble coalescence by electrolytes (see Craig et al. 1993), similar to the effect of surface active substances like SLS. This effect leads to smaller bubbles being produced, which is clearly manifested in Fig. 8c.

When using the quasi-linear model to interpret the experimental data in section 4 , the parameter $z_{\text {oofree }}$ in Eq. (13) was taken to be equal to the roughness height of an aerodynamically smooth surface. This reflects the assumption that the foam-free fraction of the water surface is aerodynamically smooth with respect to airflow. In field conditions under high winds, one can expect that it is not reasonable to model flow at the water surface as aerodynamically smooth, for example, due to the presence of ripples, that is, very steep short capillary-gravity waves. One can expect airflow separation from the crests of the ripples, which violates the assumption of an aerodynamically smooth surface. To estimate the parameter $z_{00 \text { free }}$ of the foam-free fractions of the water surface under field conditions, we made a supposition that, if there were no foam at the water surface and the wind-wave spectrum was expressed by Eq. (15) with

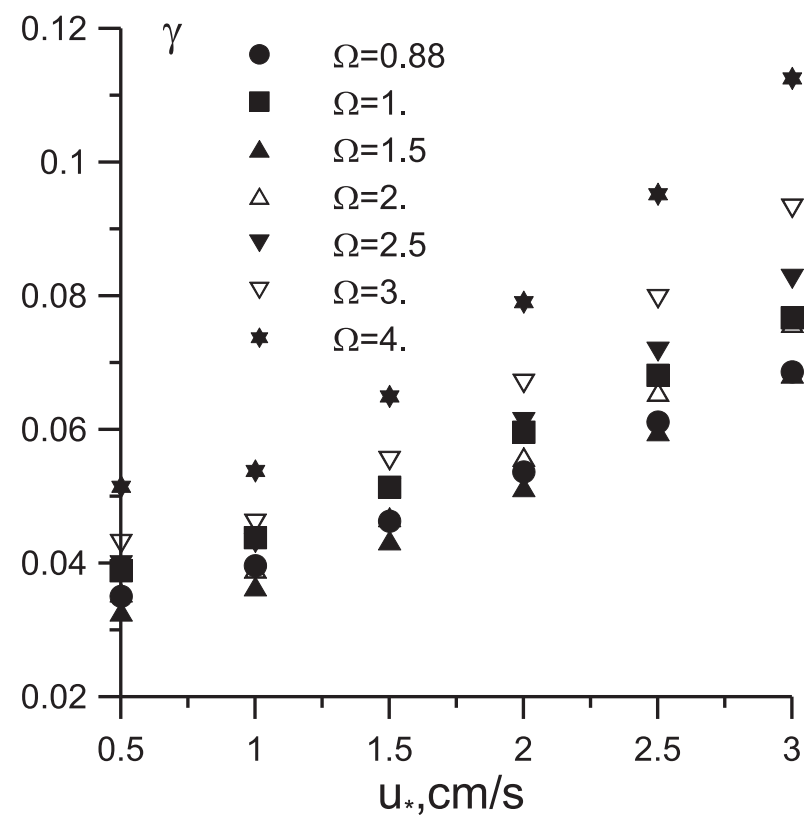

FIG. 14. Dependence of the parameter $\gamma$ in the formula for the aerodynamic roughness of the Charnock surface $z_{\text {oofree }}=\gamma z_{\mathrm{Ch}}$ vs wind friction velocity $u_{*}$ for different wave-age parameters $\Omega$. The form drag of the wind waves with the spectrum equation, Eq. (15), is calculated within the quasi-linear model of Troitskaya et al. (2012, 2014).

$C\left(S_{\text {foam }}\right)=1$, after calculations within the quasi-linear model we would get the Charnock formula for the roughness height:

$$
z_{\mathrm{Ch}}=0.0185 u_{*}^{2} / g
$$

where $g$ is acceleration under gravity and the Charnock constant is taken from Wu (1980). For brevity, we use here the term "Charnock surface" for this kind of surface.

To obtain the parameter $z_{00 \text { free }}$, we numerically solved the equations of the quasi-linear model taking the surface wave spectrum Eq. (15) where $C\left(S_{\text {foam }}\right)=1$ as the input. The iteration procedure allowed us to calculate the coefficient $\gamma$ in the equation for the aerodynamic roughness of the Charnock surface $z_{00 \text { free }}=\gamma z_{\mathrm{Ch}}$, so that the roughness height calculated within the quasi-linear model taking the form drag of the water surface into account is equal to $z_{\mathrm{Ch}}$. The dependence of $\gamma$ on the wind friction velocity for different wave-age parameters of the wave field is shown in Fig. 14. Calculations of the model with an aerodynamically smooth surface assumed in the foamfree areas with $z_{00 f r e e}$ prescribed by Eq. (14) were also performed for comparison.

The resulting calculated surface drag coefficients of a water surface partially covered by foam are 


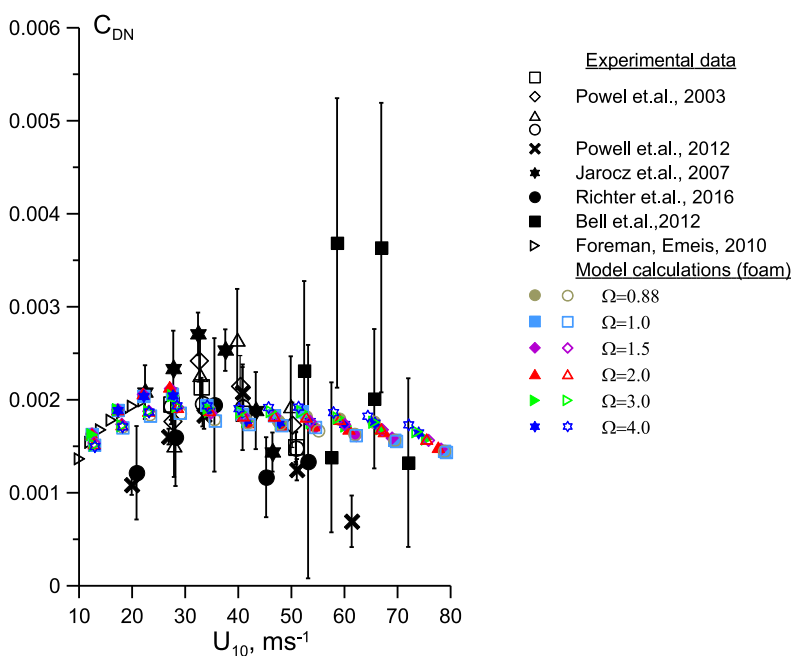

FIG. 15. Surface drag coefficient vs $10-\mathrm{m}$ wind speed. Black symbols are field data, and gray symbols are results of calculations within the quasi-linear model for different values of the wave-age parameter $\Omega$. Closed symbols are results obtained within the Charnock surface model, and open symbols are results obtained within the model with an aerodynamically smooth surface.

shown in Fig. 15. The wind velocity ranges from 10 to $80 \mathrm{~m} \mathrm{~s}^{-1}$ and the wave-age parameter $\Omega$ varies from 0.88 , corresponding to mature wind waves, to 4 . According to the data reported in Wright et al. (2001), the wave-age parameter $\Omega$ in hurricanes varies between 2.5 and 3.5. Values obtained by other authors through field data processing are also included in the same plot. It is clear from Fig. 15 that the two models of the skin roughness height of the foam-free water surface (an aerodynamically smooth surface and the Charnock surface) give similar values for the surface drag coefficient within the experimental error of $C_{D}$ and the uncertainty of the wave-age parameter under hurricane conditions. This indicates the form drag of the waves and the roughness of the foam-covered surface have a dominant effect on $C_{D}$ compared with the contribution of the foam-free fractions of the water surface. Note that Makin et al. (1995) previously mentioned the insignificance of the fraction of the highfrequency part of the surface wave spectra on the surface drag of the water surface, which prescribes the difference between the smooth and the Charnock surfaces (see Fig. 4 within that study). It is interesting to notice the peaking $C_{D}\left(U_{10}\right)$ dependence calculated within the model. This peaking is the consequence of the competing effects of an increase in wind speed on the wave form drag: a widening of the surface wave spectrum and a decrease in the slopes of short waves due to increased damping in the presence of foam, which is modeled by the factor $C\left(S_{\text {foam }}\right)$ in Eq. (15). Another factor that slightly decreases the surface drag is decreasing the size of foam bubbles taken into account by using the decreasing dependence of $\langle R\rangle$ on $u_{*}$ given by Eq. (5) in Eq. (13) for $z_{00}$. At wind speeds below $20 \mathrm{~m} \mathrm{~s}^{-1}$, the first effect dominates, because the fractional foam coverage is below $15 \%$, and then the surface drag coefficient increases with the wind speed. At higher wind speeds, the wave slope decrease due to the increase in dissipation in the presence of foam prevails, and the surface drag coefficient decreases. Notice here that the parameters of surface wave damping in the presence of foam were obtained in the laboratory experiment, and so the values may be different under field conditions.

\section{Estimate of the combined effect of foam and bag-breakup fragmentation of the air-sea interface on air-sea momentum flux under field conditions}

Along with foam at the water surface, sea spray is typical under high wind conditions. In this section, we consider the synergetic effect of foam coverage and sea spray on the aerodynamic resistance of the sea surface. It was shown by Troitskaya et al. (2017c, 2018b) that sea spray and the processes responsible for its production cause an increase in the wind tangential stress and consequently an increase in the surface drag coefficient. Recent investigations of the processes at the air-water interface at strong winds using high-speed, high-resolution video recording (see Veron et al. 2012; Troitskaya et al. 2017a,b, 2018a) have shown that bag-breakup events are the cause of the dominant spray generation mechanisms at wind speeds exceeding $20 \mathrm{~m} \mathrm{~s}^{-1}$. These events start with the appearance of a small-scale elevation of the water surface, which later transforms into a microsail and then into a membrane of water film surrounded by a thicker rim. This bursts, generating hundreds of droplets. Two "bag-breakup"-type mechanisms for spray generation are distinguished: breaking of the water film similar to the breaking of a dome of rising underwater bubbles, and fragmentation of the rim due to capillary instability. As a result, the size spectrum of droplets shows two typical scales. According to estimates, these are about 100 and $1000 \mu \mathrm{m}$, respectively. Hence, the spray generation function (SGF), which gives the number of droplets ejected from a unit area of the sea surface in a time unit, has two peaks (Troitskaya et al. 2017b, 2018a). The expression for the SGF derived by Troitskaya et al. (2017b, 2018a) is as follows: 


$$
\begin{aligned}
\frac{d F\left(r, u_{*}\right)}{d r}= & N\left(u_{*}, \Omega\right)\left\{\frac{3.3 \times 10^{-9}}{L}\left(\frac{\rho_{w} g L^{2}}{\sigma}\right)^{1.18}\left(\frac{r}{\theta}\right)^{7.3} e^{-5.2 \sinh [(3 / 7) \ln (r / \theta)]}\right. \\
& \left.+\frac{1.5 \times 10^{-4} N_{\text {rim_drops }}}{\Theta}\left(\frac{r}{\Theta}\right)^{4.5} e^{-3.94 \sinh [(1 / 2) \ln (r / \Theta)]}\right\} .
\end{aligned}
$$

Here, $\theta=0.001\left(\left\langle R_{\mathrm{bag}}\right\rangle\left(u_{*}\right)\right)^{4 / 3} L^{-1 / 3}, \Theta=0.0021\left\langle R_{\mathrm{bag}}\right\rangle\left(u_{*}\right)$, and $\left\langle R_{\mathrm{bag}}\right\rangle\left(u_{*}\right)$ is the average radius of a bag, which depends on the wind friction velocity as follows:

$$
\left\langle R_{\mathrm{bag}}\right\rangle\left(u_{*}\right)=9.6 u_{*}^{-1}
$$

$L=20 \mathrm{~m}$ is the dimensional constant derived by Lhuissier and Villermaux (2012), and $N\left(u_{*}, \Omega\right)$ is the specific number of bags per unit area per unit time, which depends on the wind and waves. Troitskaya et al. (2018a) gives two versions of this dependence on the basis of a limited laboratory dataset. The first is

$$
N\left(u_{*}, \Omega\right)=Q_{0} \frac{u_{*}^{2}}{U_{0}^{2}}\left(\frac{12.4 u_{*}^{0.5}}{g \Omega \sqrt{C_{D}}}\right)^{1.5} \exp \left(-\frac{U_{0}^{2}}{u_{*}^{2}}\right),
$$

where $U_{0}=2.00 \pm 0.13 \mathrm{~m} \mathrm{~s}^{-1}$ and $Q_{0}=9.27 \times 10^{2} \mathrm{~m}^{-4} \mathrm{~s}$ with a $95 \%$ confidence interval between $5.91 \times 10^{2} \mathrm{~m}^{-4} \mathrm{~s}$ and $1.45 \times 10^{3} \mathrm{~m}^{-4} \mathrm{~s}$.

The second is

$$
N\left(u_{*}, \Omega\right)=M_{0} \operatorname{Re}_{B}^{3 / 2} \exp \left(-\frac{M_{1}}{\operatorname{Re}_{B}^{3 / 2}}\right) .
$$

Here, $\operatorname{Re}_{B}=u_{*}^{2} / \nu_{a} \omega_{p}$ is the wind-sea Reynolds number introduced by Toba and Koga (1986), $\omega_{p}$ is the peak frequency in the wind-wave spectrum, $M_{0}=2.6 \times 10^{-4} \pm$ $0.4 \times 10^{-4} \mathrm{~m}^{-2} \mathrm{~s}^{-1}$, and $M_{1}=6.93 \times 10^{5}$ with a $95 \%$ confidence interval between $6.22 \times 10^{5}$ and $7.64 \times 10^{5}$.

We will present the results of calculations of $C_{D}$ for both variants of the SGF because, at the moment, insufficient experimental data are available with which to select a single variant.

According to Troitskaya et al. (2017c, 2018b), the contribution of bag-breakup to the air-sea momentum flux is determined by two factors. The first relates to the initial phase of these events before spay production, when bags, which are mechanical obstacles about $1 \mathrm{~cm}$ in size [for comparison, according to Powell et al. (2003) the roughness height at hurricane wind speeds is less than $2 \mathrm{~mm}$ ], significantly contribute to the aerodynamic roughness of the water surface. We use the term bag stress $F_{M b}$ for this component. The second is that the droplets acquire momentum from the airflow in the process of their formation and acceleration, producing droplet stress $F_{M d}$.
In the first case, the tangential stress caused by bags at the ocean surface, $F_{M b}$ (the fraction of the tangential force directed toward fragmentation of the water under the action of wind) is equal to the average sum of forces at individual "bags." Neglecting the inertia of a bag and considering it as a thin semispherical water film with radius $R_{\mathrm{bag}}(t)$ gives the instantaneous force on it as the superposition of the tensions at its surfaces (see details in Troitskaya et al. 2017c, 2018b):

$$
f(t)=\iint_{S} \frac{4 \sigma}{R_{\mathrm{bag}}(t)} d s=2 \pi^{2} \sigma R_{\mathrm{bag}}(t) .
$$

Parameter $F_{M b}$, meanwhile, is the product of the average number of bags $N\left(u_{*}, \Omega\right)$, Eq. (19) or Eq. (20), the bag's lifetime $\tau$, and the average force $f(t)$ during time $\tau$ :

$$
F_{M b}=4 \pi \sigma\left\langle R_{\mathrm{bag}} \tau\right\rangle N\left(u_{*}, \Omega\right)
$$

The experimental dependence of the average product $\left\langle R_{\text {bag }} \tau\right\rangle$ on $u_{*}$ obtained by Troitskaya et al. (2017c, 2018b) is as follows:

$$
\left\langle\bar{R}_{\mathrm{bag}} \tau\right\rangle=64.4 u_{*}^{-3} .
$$

As mentioned by Troitskaya et al. (2017c, 2018b), the tangential stress $F_{M b}$ depends on the specific number of bags generated in one unit of time over one unit area for the first factor and their size and lifetime for the other. These have opposite tendencies with increasing wind speed: an increase in the number of bags and a decrease in their size and lifetime. As a result, $F_{M b}$ tends to saturation with increasing wind speed.

The momentum taken up by droplets from the airflow in the process of spray production determines the droplet momentum flux $F_{M d}$. This can be estimated as the momentum acquired by droplets during their life cycle from the initial state, when they exist as a small water elevation, to the final state, when they move in the airflow, which can be measured directly from the highspeed video. The dependence of the average velocity of the droplet $u$ on the wind friction velocity was estimated by Troitskaya et al. $(2017 \mathrm{c}, 2018 \mathrm{~b})$ as $u=1.96+1.21 u_{*}$. They proposed that the spray momentum flux $F_{M d}$ defined in this way can be expressed as 


$$
F_{M d}=\rho_{w} \int_{0}^{\infty} \frac{d F\left(r, u_{*}\right)}{d r} \frac{4 \pi r^{3}}{3}\left(1.96+1.21 u_{*}\right) d r
$$

As with $F_{M b}$, the dependence of $F_{M d}$ on wind velocity is determined by opposing tendencies: on the one hand, by the increase in the number of droplets [see Eqs. (19) and (20)], and on the other hand, by the decrease in the size of droplets proportional to the mean bag size with increasing wind velocity. This results in a tendency to saturation of the function $F_{M d}\left(u_{*}\right)$, similar to $F_{M b}$. Strictly speaking, Eq. (21) gives an upper estimate for the contribution of spray to the drag at the surface because when the droplets fall, they may transfer momentum to the lower layers, resulting in acceleration of the ambient airflow.

The total shear stress in the boundary layer at hurricane winds is determined by three main factors: the form drag of the partially foam-covered water surface $F_{M s}$, the momentum flux related to spray $F_{M d}$, and aerodynamic drag of bags $F_{M b}$ :

$$
F_{M}=F_{M s}+F_{M d}+F_{M b} .
$$

For $F_{M s}$, we here use the $C_{D}\left(U_{10}\right)$ dependencies calculated within the quasi-linear model and plotted in Fig. 14. The drag coefficient in the neutrally stratified atmospheric boundary layer can be found by definition: $C_{D N}=F_{M} /\left(\rho_{a} U_{10}^{2}\right)$. The $C_{D N}\left(U_{10}\right)$ dependencies are shown in Fig. 16. We used two variants of the SGF for calculating the contribution of spray and bags, Eq. (19) and Eq. (20). The wave-age parameter $\Omega=$ $3 \pm 0.5$ in accordance with Wright et al. (2001). We also used two versions of the model of sea surface roughness: a smooth surface and the Charnock surface. Figure 16 shows that the values of $C_{D N}\left(U_{10}\right)$ from all of the models deviate by less than $20 \%$, that is, less than the error in field data. It is also interesting to notice the peaking $C_{D N}\left(U_{10}\right)$ dependence, in agreement with the results of Powell et al. (2003), Holthuijsen et al. (2012), Jarosz et al. (2007), and Richter et al. (2016). At wind speeds below $20-25 \mathrm{~m} \mathrm{~s}^{-1}$, all of the models predict a monotonous increase in the surface drag coefficient due to an increase in the form drag of the water surface and the numbers of spray droplets and bags, accompanied by insignificant suppression of waves by foam due to a low fractional foam coverage. As the wind speed increases, effects come into play that exert the opposite influence on ocean surface resistance. First, the width of the wave spectrum increases, which leads to an increase in the form drag. Second, the fractional foam coverage of the water surface increases, leading to an increase in the dissipation of the high-frequency part of the surface wave

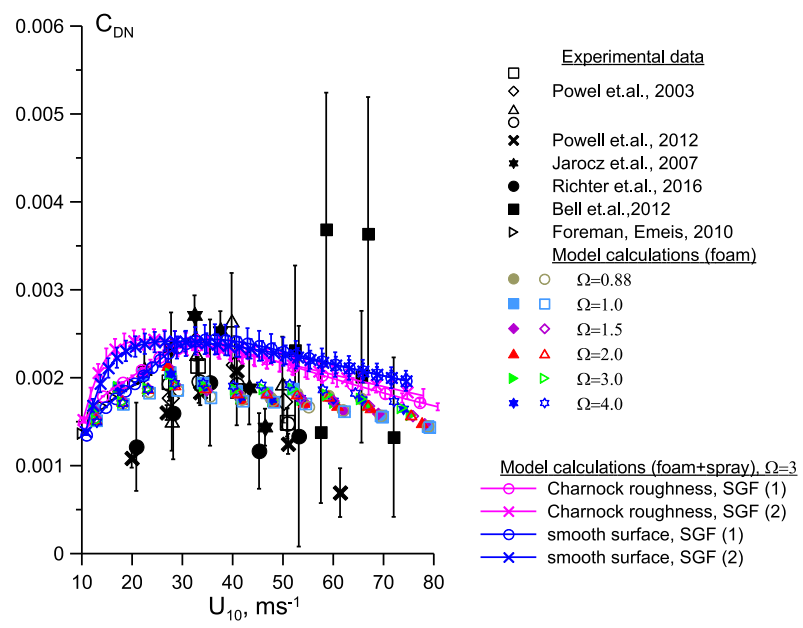

FIG. 16. Surface drag coefficient vs 10 -m wind speed. Black symbols are field data, and gray symbols are results of calculations within the quasi-linear model for different values of the wave-age parameter $\Omega$. Closed symbols are results obtained within the Charnock surface model, and open symbols are results obtained within the model with an aerodynamically smooth surface. Results of calculations when the effect of sea spray is taken into account: magenta symbols are within the Charnock surface model, blue symbols are within the model with an aerodynamically smooth surface, circles are SGF defined by Eq. (19), and crosses are SGF defined by Eq. (20).

spectrum and decreasing the surface drag. Third, the sizes of the foam bubbles decrease with an increase in the wind speed, which slightly decreases the surface drag. Fourth, the number of fragmentation events at the water surface and the spray concentration increase, which, as explained above, causes an increase in the wind shear stress. Fifth, the typical sizes and durations of fragmentation processes at the air-sea interface decrease, which reduces the elementary forcing of the airflow by droplets and bags. The combination and competition of these effects lead to unusual nonmonotonous $C_{D N}\left(U_{10}\right)$ dependencies. It should be noted that the parameters for foam and spray were obtained under laboratory conditions and most likely will be different in the real ocean. A complete dataset on the parameters of wind, waves, foam and spray in a hurricane is not yet available. The most significant difficulties are apparently the acquisition of data on the short-wave part of the surface wave spectrum, which crucially affects the aerodynamic resistance of the water surface. Another strongly uncertain factor is also sea spray. At the moment, a possible way to estimate the coefficient of aerodynamic drag in a hurricane associated with the effects of waves, foam and spray can be via adjustment of the parameters of the suggested model to the best fit of the experimental data. 


\section{Summary}

The anomalously low roughness of the sea surface at extreme winds has been the focus of a number of experimental and theoretical studies in the 15 years since seminal work by Powell et al. (2003), the discoverer of the phenomenon, was published. It has now been confirmed through numerous field (Holthuijsen et al. 2012; Jarosz et al. 2007; Richter et al. 2016) and laboratory (Donelan et al. 2004; Takagaki et al. 2012; Troitskaya et al. 2012; Takagaki et al. 2016) experiments. Theoretical explanations of the observed effect cite either peculiarities in the airflow over breaking waves (Kudryavtsev and Makin 2007; Kukulka et al. 2007) or the effect of seawater droplets and spray on wind-wave momentum exchange (Kudryavtsev 2006; Kudryavtsev and Makin 2011; Andreas 2004). The peaking dependence of sea surface drag can also be explained by peculiarities in the momentum flux from the atmosphere to the ocean in the course of sea spray production due to bag-breakup fragmentation of the air-sea interface (see Troitskaya et al. 2017c, 2018b). Alternatively, according to a recently suggested hypothesis, the surface drag reduction in hurricanes can be explained by the influence of foam covering the sea surface on its aerodynamic roughness (Golbraikh and Shtemler 2016; Holthuijsen et al. 2012).

This paper describes a series of laboratory experiments at the high-speed wind-wave flume in the Large Thermally Stratified Tank at the Institute of Applied Physics that were directed toward investing the impact of foam on the short-wave part of surface waves and on momentum exchange in the atmospheric boundary layer at high winds in the equivalent $10-\mathrm{m}$ wind speed range of $12-38 \mathrm{~m} \mathrm{~s}^{-1}$. The airflow parameters (surface drag coefficient, friction velocity, and 10-m wind speed) were retrieved from measurements of the velocity profiles. The directional frequency-wavenumber spectra of surface waves along with the integral parameters of the wave field [significant wave height, mean square slope (MSS), peak frequency and wavenumber] were retrieved from measurements of the water surface elevation obtained with a three-channel array of wave gauges. The foam on the water surface was videoed, and the fractional foam coverage and size distribution of the foam bubbles were retrieved with image processing. It was found that, at wind speeds below approximately $22-25 \mathrm{~m} \mathrm{~s}^{-1}\left(u_{*} \approx 0.9 \mathrm{~m} \mathrm{~s}^{-1}\right)$, the presence of foam increased the drag coefficient, while at higher wind speeds there was no statistically significant dependence of the drag coefficient on the presence of foam. The effect of the foam on surface wave spectra is to decrease the peak frequency and wavenumber due to damping of short waves. As a consequence, there is a noticeable decrease in the mean square slope of the water surface in comparison with a foam-free surface.

The dependence of the water surface drag coefficient on the wind velocity when foam is present can be qualitatively explained by the balance between the effect of small-scale surface roughness and the wave form-drag compared to the case of water that is free of foam. A quantitative interpretation of the effect of foam on the surface drag coefficient was performed within the quasilinear model of the atmospheric boundary layer over a waved water surface developed by Troitskaya et al. (2012, 2014). The model enables the form drag of a waved water surface to be calculated for a given friction velocity and surface wave directional spectrum and then for the roughness height or surface drag coefficient to be determined. The main physical assumption behind the model that the effects of flow separation from the wave crests is ignored. In the presence of foam at the water surface, one can expect that this assumption is valid due to its suppression of flow separation at rough surfaces (e.g., Van Dyke 1982). The quasi-linear model is based on the RANS equation, with first order closure expressed in wave-following curvilinear coordinates. Here, we use the most simple expression for the eddy viscosity coefficient, Eq. (9). The effective aerodynamic roughness $z_{00}$ is parameterized as the linear combination of the roughness heights of the foam-free and foam-covered surfaces, weighted with respect to fractional foam coverage, similarly to Golbraikh and Shtemler (2016). We emphasize the significant difference in the roughness height of the sea surface $z_{0}$ parameterized by Golbraikh and Shtemler (2016) and the effective roughness height in the eddy viscosity coefficient $z_{00}$, or skin roughness height, which does not include the form drag of the waved sea surface. The ratio between $z_{0}$ and $z_{00}$ is illustrated by Eq. (12). The skin roughness height of the foam-covered surface is assumed to be proportional to the average radius of the foam bubble, and a proportionality factor is calibrated on the basis of one of the experimental runs. In modeling the laboratory experiment, the foam-free surface is assumed to be aerodynamically smooth. Given the measured wind friction velocities and directional spectra of surface waves, we obtained surface drag coefficients in good agreement with the measured values both for the experiments using artificially generated foam and for natural foam produced due to wave breaking.

As the quasi-linear model provides a good prediction of the surface drag coefficient derived from the laboratory experiments, we used it to estimate the effect of foam on the aerodynamic resistance of the surface at high winds in a field environment. For these estimates, we used a variety of available field data on wind-wave 
spectra and fractional foam coverage. As data on the rate of suppression of the short-wave tail of the surface wave spectra in the presence of foam and the dependence of the foam bubble radius on wind speed were unavailable, these were taken from laboratory experiments. We used the spectrum provided by Donelan et al. (1985) to describe the surface waves, which is applicable at hurricane winds according to Young (2017). To express the high-frequency part of the spectrum, we used the Phillips (1958) saturation spectrum modified by the presence of foam in accordance with data from the laboratory experiments. Fractional foam coverage as a function of wind speed was taken from a paper by Holthuijsen et al. (2012) where the contributions of both whitecaps and foam streaks were taken into account. To describe the aerodynamic roughness of the foam-free fractions of the water surface under field conditions within the quasilinear model, we developed a model of a Charnock surface. Within this model, we select the roughness height of the plain surface in such a way that, in the presence of waves with the Donelan et al. (1985) spectrum for the energy-containing part of the spectrum and the Phillips (1958) saturation spectrum for the spectral tail (i.e., without foam), the roughness height of the wind flow with form drag calculated within the quasi-linear model corresponds to the Charnock equation.

The model indicates a peaking $C_{D}\left(U_{10}\right)$ dependence, which can be explained as resulting from the influence of competing factors: the amplifying effect of widening of the surface wave spectrum and the attenuating effects of a decrease of the slopes of short waves due to an increase in damping in the presence of foam and a decrease in the size of foam bubbles. Calculations with the model with an aerodynamically smooth surface assumed for foam-free areas were also performed for comparison with the Charnock surface model. Both models give similar values for the surface drag coefficient within the experimental error of measuring $C_{D}$ and the uncertainty of the wave-age parameter under hurricane conditions. This indicates that the form drag of the waves and the roughness of the foam-covered surface have a dominant effect on $C_{D}$ compared with the contribution of the foam-free fractions of the water surface.

We also estimate the synergetic effect of the foam coverage and the phenomenon of bag-breakup fragmentation of the air-sea interface (see Veron et al. 2012; Troitskaya et al. 2017a,b, 2018a) on the aerodynamic resistance of the water surface in the field environment. According to Troitskaya et al. (2017c, 2018b), the contribution of bag-breakup fragmentation to the surface drag coefficient is a nonmonotonous function of wind velocity due to two competing effects: first, an increase in the number of fragmentation events and the concentration of spray and second, a decrease in the typical periodicity of fragmentation and the sizes of the fragments produced, reducing their elementary forcing effect on the airflow. The resulting $C_{D}\left(U_{10}\right)$ dependence, which combines the effects of foam coverage and bag-breakup fragmentation, appears to be an unusual nonmonotonous function. It should be noted that the parameters of foam and spray were obtained under laboratory conditions and may be different in the real ocean. However, it can be expected that a combination of various complex factors typical for strong wind conditions, such as waves and spray, will have a significant effect on the aerodynamic resistance of the ocean surface and lead to an unusual dependence of the coefficient of aerodynamic drag on wind speed as predicted by our simplified analysis.

Acknowledgments. This work has been supported by the Russian Science Foundation (Project 14-17-00667), the Seventh Framework Programme (Project PIRSESGA-2013-612610), and the Russian Foundation for Basic Research (Projects 16-05-00839, 18-55-50005, 18-05-60299, 18-35-20068, and 19-05-00249). Sergej Zilitinkevich additionally acknowledges support from the Academy of Finland project ClimEco 314 798/799; Alexander Kandaurov acknowledges support by the President grant for young scientists MC-2041.2017.5 and Russian Science Foundation (Project 18-77-00074). The basic salary of the authors from IAP is financed by government contract 0035-20140032. The experiments were performed at the Unique Scientific Facility "Complex of Large-Scale Geophysical Facilities" (http://www.ckp-rf.ru/usu/77738/). Maxim Vdovin acknowledges support by the President grant for young scientists SP-1740.2016.1. The authors thank Ms. Rebecca Thompson for improving the use of English and in organization of the text.

\section{REFERENCES}

Andreas, E. L, 2004: Spray stress revisited. J. Phys. Oceanogr., 34, 1429-1440, https://doi.org/10.1175/1520-0485(2004)034<1429: $\mathrm{SSR}>2.0 . \mathrm{CO} ; 2$.

—_, and K. A. Emanuel, 2001: Effects of sea spray on tropical cyclone intensity. J. Atmos. Sci., 58, 3741-3751, https://doi.org/ 10.1175/1520-0469(2001)058<3741:EOSSOT >2.0.CO;2.

_ L. Mahrt, and D. Vickers, 2012: A new drag relation for aerodynamically rough flow over the ocean. J. Atmos. Sci., 69, 2520-2537, https://doi.org/10.1175/JAS-D-11-0312.1.

Bagnold, R. A., 1941: The Physics of Blown Sand and Desert Dunes. Methuen, $265 \mathrm{pp}$.

Barenblatt, G., and G. Golitsyn, 1974: Local structure of mature dust storms. J. Atmos. Sci., 31, 1917-1933, https://doi.org/10.1175/ 1520-0469(1974)031<1917:LSOMDS > 2.0.CO;2.

Belcher, S. E., T. M. J. Newley, and J. C. R. Hunt, 1993: The drag on an undulating surface induced by the flow of a turbulent boundary layer. J. Fluid Mech., 249, 557-598, https://doi.org/ 10.1017/S0022112093001296. 
Bell, M. M., M. T. Montgomery, and K. A. Emanuel, 2012: Air-sea enthalpy and momentum exchange at major hurricane wind speeds observed during CBLAST. J. Atmos. Sci., 69, $3197-$ 3222, https://doi.org/10.1175/JAS-D-11-0276.1.

Bender, M. A., and I. Ginis, 2000: Real-case simulations of hurricaneocean interaction using a high-resolution coupled model: Effects on hurricane intensity. Mon. Wea. Rev., 128, 917-946, https:// doi.org/10.1175/1520-0493(2000)128<0917:RCSOHO >2.0.CO;2.

Bister, M., and K. A. Emanuel, 1998: Dissipative heating and hurricane intensity. Meteor. Atmos. Phys., 65, 233-240, https:// doi.org/10.1007/BF01030791.

Black, P. G., and Coauthors, 2007: Air-sea exchange in hurricanes: Synthesis of observations from the coupled boundary layer air-sea transfer experiment. Bull. Amer. Meteor. Soc., 88, 357374, https://doi.org/10.1175/BAMS-88-3-357.

Buckley, M. P., and F. Veron, 2016: Structure of the airflow above surface waves. J. Phys. Oceanogr., 46, 1377-1397, https://doi.org/ 10.1175/JPO-D-15-0135.1.

Craig, V. S. J., B. W. Ninham, and R. M. Pashley, 1993: The effect of electrolytes on bubble coalescence in water. J. Phys. Chem., 97, 10 192-10 197, https://doi.org/10.1021/j100141a047.

Donelan, M., J. Hamilton, and W. H. Hui, 1985: Directional spectra of wind generated waves. Philos. Trans. Roy. Soc. London, 315A, 509-562, https://doi.org/10.1098/rsta.1985.0054.

— W. M. Drennan, and A. K. Magnusson, 1996: Non-stationary analysis of the directional properties of propagating waves. J. Phys. Oceanogr., 26, 1901-1914, https://doi.org/10.1175/15200485(1996)026<1901:NAOTDP > 2.0.CO;2.

—_, B. K. Haus, N. Reul, W. J. Plant, M. Stiassnie, H. C. Graber, O. B. Brown, and E. S. Saltzman, 2004: On the limiting aerodynamic roughness of the ocean in very strong winds. Geophys. Res. Lett., 31, L18306, https://doi.org/10.1029/ 2004GL019460.

Druzhinin, O. A., Yu. I. Troitskaya, and S. S. Zilitinkevich, 2012: Direct numerical simulation of a turbulent wind over a wavy water surface. J. Geophys. Res., 117, C00J05, https://doi.org/ 10.1029/2011JC007789.

Emanuel, K. A., 1986: An air-sea interaction theory for tropical cyclones. Part I: Steady-state maintenance. J. Atmos. Sci., 43, 585-605, https://doi.org/10.1175/1520-0469(1986)043<0585: AASITF $>2.0 . \mathrm{CO} ; 2$.

Fairall, C. W., E. F. Bradley, J. E. Hare, A. A. Grachev, and J. B. Edson, 2003: Bulk parameterization of air-sea fluxes: Updates and verification for the COARE algorithm. J. Climate, 16, 571-591, https://doi.org/10.1175/1520-0442(2003)016<0571: BPOASF $>2.0 . \mathrm{CO} ; 2$.

Falkovich, A. I., A. P. Kain, and I. Ginis, 1995: The influence of airsea interaction on the development and motion of a tropical cyclone: Numerical experiments with a triply nested model. Meteor. Atmos. Phys., 55, 167-184, https://doi.org/10.1007/ BF01029825.

Foreman, R. J., and S. Emeis, 2010: Revisiting the definition of the drag coefficient in the marine atmospheric boundary layer. J. Phys. Oceanogr., 40, 2325-2332, https://doi.org/10.1175/ 2010JPO4420.1.

Garratt, J. R., 1977: Review of drag coefficients over oceans and continents. Mon. Wea. Rev., 105, 915-929, https://doi.org/ 10.1175/1520-0493(1977)105<0915:RODCOO > 2.0.CO;2.

Golbraikh, E., and Y. M. Shtemler, 2016: Foam input into the drag coefficient in hurricane conditions. Dyn. Atmos. Oceans, 73, 1-9, https://doi.org/10.1016/j.dynatmoce.2015.10.005.

Hinze, J. O., 1959: Turbulence: An Introduction to its Mechanism and Theory. McGraw-Hill, 586 pp.
Holthuijsen, L. H., M. D. Powell, and J. D. Pietrzak, 2012: Wind and waves in extreme hurricanes. J. Geophys. Res., 117, C09003, https://doi.org/10.1029/2012JC007983.

IAP RAS, 2018: Thermo-stratified wind-wave pool. Accessed 3 January 2019, http://unu.ipfran.ru/pool.

Janssen, P. A. E. M., 1989: Wave-induced stress and the drag of air flow over sea waves. J. Phys. Oceanogr., 19, 745-754, https://doi.org/10.1175/1520-0485(1989)019<0745:WISATD > 2.0.CO;2.

Jarosz, E., D. A. Mitchell, D. W. Wang, and W. J. Teague, 2007: Bottom-up determination of air-sea momentum exchange under a major tropical cyclone. Science, 315, 1707-1709, https://doi.org/ 10.1126/science.1136466.

Kandaurov, A. A., Yu. I. Troitskaya, D. A. Sergeev, M. I. Vdovin, and G. A. Baidakov, 2014: Average velocity field of the air flow over the water surface in a laboratory modeling of storm and hurricane conditions in the ocean. Izv., Atmos. Ocean. Phys., 50, 399, https://doi.org/10.1134/S000143381404015X.

Komen, G. J., L. Cavaleri, M. Donelan, K. Hasselmann, S. Hasselmann, and P. A. E. M. Janssen, 1994: Dynamics and Modelling of Ocean Waves. Cambridge University Press, $532 \mathrm{pp}$.

Kudryavtsev, V. N., 2006: On the effect of sea drops on the atmospheric boundary layer. J. Geophys. Res., 111, C07020, https:// doi.org/10.1029/2005JC002970.

— surface at high winds. Bound.-Layer Meteor., 125, 289-303, https://doi.org/10.1007/s10546-007-9184-7.

— and - 2011: Impact of ocean spray on the dynamics of the marine atmospheric boundary layer. Bound.-Layer Meteor., 140, 383-410, https://doi.org/10.1007/s10546-011-9624-2.

Kukulka, T., T. Hara, and S. E. Belcher, 2007: A model of the air-sea momentum flux and breaking wave distribution for strongly forced wind-waves. J. Phys. Oceanogr., 37, 1811-1828, https:// doi.org/10.1175/JPO3084.1.

Large, W. G., and S. Pond, 1981: Open ocean momentum flux measurements in moderate to strong winds. J. Phys. Oceanogr., 11, 324-336, https://doi.org/10.1175/1520-0485(1981)011<0324: OOMFMI $>2.0 . \mathrm{CO} ; 2$.

Lhuissier, H., and E. Villermaux, 2012: Bursting bubble aerosols. J. Fluid Mech., 696, 5-44, https://doi.org/10.1017/jfm.2011.418.

Makin, V. K., 2005: A note on drag of the sea surface at hurricane winds. Bound.-Layer Meteor., 115, 169-176, https://doi.org/ 10.1007/s10546-004-3647-x.

_ V. N. Kudryavtsev, and C. Mastenbroek, 1995: Drag of the sea surface. Bound.-Layer Meteor., 73, 159-182, https://doi.org/ 10.1007/BF00708935.

Phillips, O. M., 1958: The equilibrium range in the spectrum of wind generated water waves. J. Fluid Mech., 4, 426-434, https://doi.org/ 10.1017/S0022112058000550.

Powell, M. D., P. J. Vickery, and T. A. Reinhold, 2003: Reduced drag coefficient for high wind speeds in tropical cyclones. Nature, 422, 279-283, https://doi.org/10.1038/nature01481.

Reul, N., H. Branger, and J.-P. Giovanangeli, 1999: Air flow separation over unsteady breaking waves. Phys. Fluids, 11, 19591961, https://doi.org/10.1063/1.870058.

Richter, D. H., R. Bohac, and D. P. Stern, 2016: An assessment of the flux profile method for determining air-sea momentum and enthalpy fluxes from dropsonde data in tropical cyclones. J. Atmos. Sci., 73, 2665-2682, https://doi.org/10.1175/ JAS-D-15-0331.1.

Shtemler, Y. M., E. Golbraikh, and M. Mond, 2010: Wind-wave stabilization by a foam layer between the atmosphere and the ocean. Dyn. Atmos. Oceans, 50, 1-15, https://doi.org/10.1016/ j.dynatmoce.2009.08.002. 
Smol'yakov, A. V., 1973: Spectrum of the quadrupole radiation of a planar turbulent boundary layer. Akust. Zh., 19, 420-425.

Soloviev, A. V., R. Lukas, M. Donelan, B. K. Haus, and I. Ginis, 2014: The air-sea interface and surface stress under tropical cyclones. Sci. Rep., 4, 5306, https://doi.org/10.1038/srep05306.

Stauffer, C. E., 1965: The measurement of surface tension by the pendant drop technique.J. Phys. Chem., 69, 1933-1938, https://doi.org/10.1021/j100890a024.

Takagaki, N., S. Komori, N. Suzuki, K. Iwano, T. Kuramoto, S. Shimada, R. Kurose, and K. Takahashi, 2012: Strong correlation between the drag coefficient and the shape of the wind sea spectrum over a broad range of wind speeds. Geophys. Res. Lett., 39, L23604, https://doi.org/10.1029/2012GL053988.

,,,,---- and R. Kurose, 2016: Mechanism of drag coefficient saturation at strong wind speeds. Geophys. Res. Lett., 43, 9829-9835, https://doi.org/10.1002/2016GL070666.

Taylor, P. K., and M. J. Yelland, 2001: The dependence of sea surface roughness on the height and steepness of the waves. J. Phys. Oceanogr., 31, 572-590, https://doi.org/10.1175/1520-0485(2001) 031<0572:TDOSSR > 2.0.CO;2.

Toba, Y., and M. Koga, 1986: A parameter describing overall conditions of wave breaking, whitecapping, sea-spray production and wind stress. Oceanic Whitecaps, E.C. Monahan and G. MacNiocaill, Eds., D. Reidel, 37-47.

Troitskaya, Yu. I., and G. V. Rybushkina, 2008: Quasi-linear model of interaction of surface waves with strong and hurricane winds. Izv., Acad. Sci., USSR, Atmos. Oceanic Phys., 44, 621645, https://doi.org/10.1134/S0001433808050083.

— - D. Sergeev, O. Ermakova, and G. Balandina, 2011: Statistical parameters of the air turbulent boundary layer over steep water waves measured by the PIV technique. J. Phys. Oceanogr., 41, 1421-1454, https://doi.org/10.1175/2011JPO4392.1.

,-- A. A. Kandaurov, G. A. Baidakov, M. A. Vdovin, and V. I. Kazakov, 2012: Laboratory and theoretical modeling of air-sea momentum transfer under severe wind conditions. J. Geophys. Res., 117, C00J21, https://doi.org/10.1029/2011JC007778.

,-- , O. Druzhinin, A. A. Kandaurov, O. S. Ermakova, E. V. Ezhova, I. Esau, and S. Zilitinkevich, 2014: Atmospheric boundary layer over steep surface waves. Ocean Dyn., 64, 1153-1161, https://doi.org/10.1007/s10236-014-0743-4.

—- A. Kandaurov, O. Ermakova, D. Kozlov, D. Sergeev, and S. Zilitinkevich, 2017a: Bag-breakup fragmentation as the dominant mechanism of sea-spray production in high winds. Sci. Rep., 7, 1614, https://doi.org/10.1038/s41598-017-01673-9. —, O. S. Ermakova, A. A. Kandaurov, D. S. Kozlov, D. A. Sergeev, and S. S. Zilitinkevich, 2017b: Fragmentation of the "bag-breakup" type as a mechanism of the generation of sea spray at strong and hurricane winds. Dokl. Earth Sci., 477, 1330-1335, https://doi.org/10.1134/S1028334X17110174.

,,,,$---- \ldots$, and,$- 2017 \mathrm{c}$ : Non-monotonous dependence of the ocean surface drag coefficient on the hurricane wind speed due to the fragmentation of the ocean-atmosphere interface. Dokl. Earth Sci., 477, 1373-1378, https://doi.org/ 10.1134/S1028334X17110265.

—- O. Druzhinin, D. Kozlov, and S. Zilitinkevich, 2018a: "Bag-breakup" spume droplet generation mechanism at hurricane wind. Part I: Spray generation function. J. Phys. Oceanogr., 48, 2167-2188, https://doi.org/10.1175/JPO-D17-0104.1.

$-, \ldots,-$, and $—, 2018 \mathrm{~b}$ : "Bag-breakup" spume droplet generation mechanism at hurricane wind. Part II: Contribution to momentum and enthalpy transfer. J. Phys. Oceanogr., 48, 2189-2207, https://doi.org/10.1175/JPO-D-170105.1.

Van Dyke, M., 1982: An Album of Fluid Motion. 10th ed. Parabolic Press, 175 pp.

Veron, F., C. Hopkins, E. L. Harrison, and J. A. Mueller, 2012: Sea spray spume droplet production in high wind speeds. Geophys. Res. Lett., 39, L16602, https://doi.org/10.1029/ 2012 GL052603.

Wright, C. W., and Coauthors, 2001: Hurricane directional wave spectrum spatial variation in the open ocean. J. Phys. Oceanogr., 31, 2472-2488, https://doi.org/10.1175/1520-0485(2001) 031<2472:HDWSSV $>2.0 . \mathrm{CO} ; 2$.

Wu, J., 1980: Wind-stress coefficients over sea surface near neutral conditions-A Revisit. J. Phys. Oceanogr., 10, 727-740, https://doi.org/10.1175/1520-0485(1980)010<0727:WSCOSS > 2.0. $\mathrm{CO} ; 2$

Yang, D., and L. Shen, 2010: Direct-simulation-based study of turbulent flow over various waving boundaries. J. Fluid Mech., 650, 131-180, https://doi.org/10.1017/S0022112009993557.

Young, I. R., 2017: A review of parametric descriptions of tropical cyclone wind-wave generation. Atmosphere, 8, 194, https:// doi.org/10.3390/atmos8100194. 